CERN-TH/2000-269, FTUAM-00/21, IFT-UAM/CSIC-00-27

hep-th/0009135

\title{
Type IIB Orientifolds without Untwisted Tadpoles, and non-BPS D-branes
}

\author{
R. Rabadán ${ }^{1}$, A. M. Uranga ${ }^{2}$ \\ ${ }^{1}$ Dpto. Física Teórica, Universidad Autónoma de Madrid \\ 28049 Cantoblanco, Madrid, Spain \\ 2 Theory Division, CERN \\ CH-1211 Geneva 23, Switzerland
}

\begin{abstract}
We discuss the construction of six- and four-dimensional Type IIB orientifolds with vanishing untwisted $R R$ tadpoles, but generically non-zero twisted RR tadpoles. Tadpole cancellation requires the introduction of D-brane systems with zero untwisted RR charge, but non-zero twisted RR charges. We construct explicit models containing branes and antibranes at fixed points of the internal space, or non-BPS branes partially wrapped on it. The models are non-supersymmetric, but are absolutely stable against decay to supersymmetric vacua. For particular values of the compactification radii tachyonic modes may develop, triggering phase transitions between the different types of non-BPS configurations of branes, which we study in detail in a particular example. As an interesting spin-off, we show that the $\mathbf{T}^{\mathbf{6}} / \mathbf{Z}_{\mathbf{4}}$ orientifold without vector structure, previously considered inconsistent due to uncancelable twisted tadpoles, can actually be made consistent by introducing a set of brane-antibrane pairs whose twisted charge cancels the problematic tadpole.
\end{abstract}




\section{Introduction}

Type IIB orientifolds [1, 2, 6, 团 (see [5, 6, 0, 8, 9, 10, 11] for more recent references) allow the construction of large classes of supersymmetric string vacua, which have played a prominent role e.g. in the study of string duality. Recently, and partially motivated by the new developments in non-supersymmetric strings and non-supersymmetric states in string theory (see [12, 13] for a review) and their relation to brane-antibrane configurations, it has become natural to incorporate antibranes in the construction of

non-supersymmetric type IIB orientifolds [14, 15, 16] (see also [17, 18, 19] for further developments), thus enlarging the class of models available through this construction. Interestingly enough, such formal developments have led to a breakthrough in the construction of phenomenologically realistic orientifold models [17, 20].

Another interesting advantage in allowing the introduction of antibranes in the orientifold construction is that they can be used to cancel RR tadpoles in models for which no supersymmetry-preserving solution exists. A prototypical example is provided by models with untwisted tadpoles whose cancellation requires a net negative D-brane charge. Six-dimensional [15, [16] and four-dimensional [18] examples of this type are easily obtained by considering a particular choice of the orientifold projection on twisted sectors (sometimes referred to in the literature [21, 22, 23] as 'with vector structure'), whose untwisted crosscap tadpole requires a positive net number of antibranes for consistency.

In this paper we study another class of type IIB orientifolds whose crosscap tadpoles cannot be canceled by using just D-branes, i.e. preserving supersymmetry. The models contain non-zero twisted RR tadpoles, but zero untwisted RR tadpoles. To render them consistent one needs to introduce systems of D-branes contributing to twisted tadpoles, but with zero charge under the untwisted RR forms. Such brane systems are necessarily non-supersymmetric. Two simple examples are for instance sets of branes and antibranes sitting at fixed points in the internal space [16] (see e.g. [24, 25] for such configurations in the context of non-BPS states in string theory), or non-BPS branes passing through fixed points (the so-called truncated branes [26]).

Since no supersymmetric solution to the tadpole conditions exists, these models are absolutely stable against decay to supersymmetric configurations. However, a given non-supersymmetric configuration of branes satisfying the tadpole conditions may decay to a different consistent (and non-supersymmetric as well) configuration as some of the geometric moduli are varied. The space of geometries splits into different phases, at each of which at least one (and possibly several) of these configurations of branes 
is stable and tachyon-free. At their boundaries some configurations become unstable and decay, leading to interesting phase transitions in which the low-energy field theory changes drastically. This situation is analogous to that studied in the literature on non-BPS states in string theory [24, 25, 12, 13], and has been considered in the orientifold context in 19 .

In this paper we present several explicit six- and four-dimensional orientifolds with the above features, where the tadpoles are cancelled using brane-antibrane pairs at orbifold fixed points. Tadpoles could also be cancelled by the introduction of a suitable set of non-BPS D-branes. In a particular six-dimensional example, we construct explicitly the set of possible configurations of branes cancelling the tadpoles, and discuss their stability regimes as the compactification radii are varied, leading to a picture of the phase diagram for this model.

In [19], an asymmetric orientifold model without untwisted tadpoles but with twisted tadpoles was constructed, and several of these issues were addressed. Our purpose in this paper is to illustrate the construction of orientifolds without untwisted tadpoles in the context of completely left-right symmetric constructions. We hope that, since they admit a geometric interpretation, the class of models we consider will allow a more intuitive and systematic study of this type of string vacua.

Our investigation leads to an interesting insight into an old problem in the orientifold context. The $\mathbf{T}^{\mathbf{6}} / \mathbf{Z}_{\mathbf{4}}$ orientifold without vector structure contains a non-zero twisted tadpole with an unusual volume dependence, which cannot be cancelled by the D9- or D5 $5_{3}$-branes 1 present in the model (required by the existence of untwisted tadpoles) [9]. Therefore it has been considered that the model admits no solution to the tadpole cancellation conditions, and hence is inconsistent. Our analysis shows that the latter claims are actually incorrect. In particular, we show that it is possible to add configurations of branes which cancel the problematic twisted tadpole, while give zero contribution to the untwisted tadpoles (specifically pairs of $\mathrm{D} 5_{1}-\overline{\mathrm{D}}_{1}$ and/or $\mathrm{D} 5_{2^{-}}$ $\overline{\mathrm{D}}_{2}$ branes). In this way, we achieve the construction of explicit consistent compact $\mathbf{T}^{\mathbf{6}} / \mathbf{Z}_{\mathbf{4}}$ orientifolds where all tadpoles are cancelled. The correct statement about the $\mathbf{Z}_{4}$ orientifold without vector structure is then that it contains a twisted tadpole, whose cancellation requires its own new set of branes contributing to the twisted tadpole, but not to the untwisted tadpoles. Notice that our point is different from that made in [18, 10], where the $\mathbf{T}^{\mathbf{6}} / \mathbf{Z}_{\mathbf{4}}$ orientifold studied is actually different from that in [9], since it corresponds to a projection with vector structure, for which the problematic tadpole

\footnotetext{
${ }^{1}$ In our notation, $\mathrm{D} 5_{i}$-branes wrap the $i^{\text {th }}$ complex plane, while $\mathrm{D} 7_{i}$-branes are transverse to it. We also denote antibranes with a bar.
} 
simply vanishes.

The $\mathbf{T}^{\mathbf{6}} / \mathbf{Z}_{\mathbf{4}}$ model (and other four-dimensional models with analogous features, like the $\mathbf{Z}_{\mathbf{8}}, \mathbf{Z}_{\mathbf{8}}^{\prime}, \mathbf{Z}_{\mathbf{1 2}}^{\prime}$ [9]) is similar to the orientifolds without untwisted tadpoles mentioned above, in the following sense. Both types of models contain twisted tadpoles whose cancellation requires the introduction of branes for which no untwisted tadpole exists. We hope this note clarifies that such models can be consistently completed by appropriate sets of D-branes, and illustrates the power of brane-antibrane systems (or other non-BPS configurations of branes) in making problematic models consistent.

The paper is organized as follows. In sections 2,3 , and 4 we study the construction of certain families of type IIB orientifolds without untwisted tadpoles. The general pattern in their construction is the following: We consider type IIB theory compactified on a toroidal orbifold, $\mathbf{T}^{\mathbf{4}} / \boldsymbol{\Gamma}$ or $\mathbf{T}^{\mathbf{6}} / \boldsymbol{\Gamma}$ (even though a more general starting point, like a general CY or a non-geometric CFT compactification, is conceivable). We mod out the theory by an action $\Omega g$, where $\Omega$ is world-sheet parity, and $g$ is a geometric action with $g^{2} \in \Gamma$ (required by closure). Equivalently, we mod out type IIB theory by the orientifold group $G_{\text {orient }}$, generated by $\Gamma$ and $\Omega g$. An element $\Omega h$ in the orientifold group generates a tadpole (via a crosscap diagram) at $h$-fixed points for a $R R$ field in the $h^{2}$-twisted sector. Hence, to obtain models without untwisted tadpoles, we need $G_{\text {orient }}$ to contain no elements $\Omega h$ with an order two $h$.

In Sections 2, 3 we consider a simple realization of this idea. We consider a $\mathbf{Z}_{\mathbf{2 N}}$ action $\alpha$ acting crystallographically on $\mathbf{T}^{4}$ or $\mathbf{T}^{6}$, and consider type IIB theory on $\mathbf{T}^{\mathbf{6}} / \mathbf{Z}_{\mathbf{N}}$, with $\mathbf{Z}_{\mathbf{N}}$ generated by $\theta=\alpha^{2}$. We further mod out this theory by $\Omega \alpha$, hence the orientifold group has the structure

$$
G_{\text {orient }}=\left\{1, \Omega \alpha, \alpha^{2}, \ldots, \Omega \alpha^{2 k-1}, \alpha^{2 k}, \ldots\right\}
$$

For odd $N, \alpha^{N}$ is of order two, and the orientifold group element $\Omega \alpha^{N}$ leads to untwisted tadpoles. The resulting models are therefore T-dual to familiar models studied in the literature. On the other hand, for even $N, \Omega$ is never accompanied by an order two action, and the resulting models contain no untwisted tadpoles, leading to new type IIB orientifolds. For some models there is an additional (accidental) cancellation of twisted tadpoles, resulting in a set of models which do not require open string sectors for consistency, i.e. a set of consistent unoriented closed string theories. The generic case, however, corresponds to models with non-zero twisted tadpoles, which we cancel through the introduction of brane-antibrane pairs at fixed points in the internal space. We construct several six-dimensional (Section 2) and four-dimensional (Section 3) examples of this type. 
In Section 4 we consider a further possibility to build new six-dimensional orientifolds without untwisted tadpoles, based on a different structure of the orientifold group. The models are obtained by modding out $\mathbf{T}^{4} / \mathbf{Z}_{\mathbf{N}}$, for even $N$, by the orientifold action $\Omega \Pi$, with $\Pi:\left(z_{1}, z_{2}\right) \rightarrow\left(z_{2},-z_{1}\right)$.

In Section 5 we discuss tadpole cancellation in the $\mathbf{T}^{\mathbf{6}} / \mathbf{Z}_{\mathbf{4}}$ orientifold without vector structure of [9], and provide a consistent solution to the tadpole conditions (including the problematic twisted tadpole) using $\mathrm{D} 5_{1}-\overline{\mathrm{D}}_{1}$ pairs, in addition to the usual D9- and D5 $5_{3}$-branes.

In Section 6 we describe the stability regions of different brane configurations for the particular six-dimensional orientifold of $\mathbf{T}^{\mathbf{4}} / \mathbf{Z}_{\mathbf{2}}$ studied in Section 2, and obtain the phase diagram for this model. This Section can be read independently of Sections 3,4 and 5. Section 7 contains our final conclusions.

\section{Six-dimensional examples}

Before considering the more involved case of four-dimensional models, it will be convenient to make the above ideas explicit in the six-dimensional context. In this section we consider orientifolds of type IIB theory compactified on $\mathbf{T}^{\mathbf{4}} / \mathbf{Z}_{\mathbf{N}}$. The orientifold action is $\Omega^{\prime} \alpha$, where $\Omega^{\prime}=\Omega R$, and $R$ reflects all compact directions 2 . Also, $\alpha$ is a $\mathbf{Z}_{\mathbf{2 N}}$ crystallographic action on $\mathbf{T}^{4}$, and $\theta=\alpha^{2}$ generates the orbifold group $\mathbf{Z}_{\mathbf{N}}$. Following the general arguments in the introduction, such orientifolds produce zero untwisted tadpoles when $N$ is even, so the only crystallographically consistent $\alpha$ is the $\mathbf{Z}_{\mathbf{4}}$ twist $\alpha:\left(z_{1}, z_{2}\right) \rightarrow\left(e^{2 \pi i \frac{1}{4}} z_{1}, e^{-2 \pi i \frac{1}{4}} z_{2}\right)$.

This type of models was studied in the non-compact context (i.e. orientifolds of $\mathbf{C}^{2} / \mathbf{Z}_{\mathbf{N}}$, where the crystallographic constraint can be dropped) in [27, whose basic results can be used in the compact context as well. One important difference is that in the non-compact case the cancellation of untwisted charge may be violated, since the corresponding flux may escape to infinity through the non-compact transverse dimensions. Hence, models in [27] used D5-branes to cancel twisted tadpoles, but did not include $\overline{\mathrm{D} 5}$-branes to compensate for their untwisted charge. Our compact models will however necessarily contain equal numbers of D5- and $\overline{\mathrm{D} 5}$-branes. A minor difference is that [27 considered a T-dual version of these models, with orientifold action $\Omega^{\prime} R_{3}(-)^{F_{L}}$, in which the crosscap contributions to the tadpoles are reduced by a factor of four with respect to our models here.

As discussed in [27] and following [22, 23], it is important to notice the existence

\footnotetext{
${ }^{2}$ Choosing other orientifold actions, like $\Omega \alpha$, leads merely to T-dual versions of our models.
} 
of two orientifold projections for even $N, N=2 P$, which we refer to as $\mathbf{A}$ - and Bprojections, which differ in their action on order two twisted sectors, and which is correlated [22] with the choice of vector structure on the D-brane Chan-Paton bundle [21. We discuss them in turn.

\subsection{Models with A-type projection}

The first type of projection, which we denote as A-type, chooses left-right symmetric (antisymmetric) states in the NS-NS (RR) order two twisted sector. The closed string twisted sector at a $\mathbf{C}^{2} / \mathbf{Z}_{\mathbf{N}}$ point leads to $P$ hyper- and $P-1$ tensor multiplets of $D=6 \mathcal{N}=1$ supersymmetry. When open string sectors are required, this projection is correlated with a choice of Chan-Paton matrices without vector structure of the form (A.1) for D5- and D9-branes (and antibranes), and orientifold actions (A.2) for D5-branes and (A.3) for D9-branes.

Evaluation of the relevant amplitudes leads to the following crosscap twisted tadpoles [27] at each $\mathbf{C}^{2} / \mathbf{Z}_{\mathbf{N}}$ orientifold point

$$
\mathcal{T}_{\theta^{k}}=+32 \delta_{k, 1 \bmod 2} \cos (\pi k / N) \quad k=1, \ldots, N-1
$$

In this and similar formulae, we normalize these twisted orientifold charges in units in which the D5- and D9-brane twisted disk tadpole are $4 \sin (\pi k / N) \operatorname{Tr} \gamma_{\theta^{k}, 5}$ and $\operatorname{Tr} \gamma_{\theta^{k}, 9}$, respectively. However, we prefer not to introduce disk contributions for the moment, since in some cases open string sectors may not be required for consistency.

For the case of interest in the compact setup, the $\Omega^{\prime} \alpha$ orientifold of $\mathbf{T}^{\mathbf{4}} / \mathbf{Z}_{\mathbf{2}}$, the above closed string tadpoles vanish, and the theory is consistent without the introduction of open string sectors. In fact this model has already appeared in [6]. The untwisted closed string sector contains the $D=6 \mathcal{N}=1$ supergravity multiplet, the dilaton tensor multiplet, and two hyper- and two tensor multiplets. In the closed twisted sectors, the four $\alpha$-invariant $\mathbf{Z}_{\mathbf{2}}$ fixed point give one hypermultiplet each, while the remaining twelve $\mathbf{Z}_{\mathbf{2}}$ fixed points (paired by $\Omega^{\prime} \alpha$ ) give a total of six hyper- and six tensor multiplets each. The spectrum, shown in Table 1, is free of gravitational anomalies.

\begin{tabular}{|c||c|}
\hline Untwisted & $\mathcal{N}=1$ SUGRA $+3 \mathbf{T}+2 \mathbf{H}$ \\
\hline Twisted & $6 \mathbf{T}+10 \mathbf{H}$ \\
\hline
\end{tabular}

Table 1: Spectrum of the $\Omega^{\prime} \alpha$ orientifold of $\mathbf{T}^{\mathbf{4}} / \mathbf{Z}_{\mathbf{2}}$ with $\mathbf{A}$-type projection. 


\subsection{Models with B-type projection}

The second type of projection, which we denote as $\mathbf{B}$, chooses left-right antisymmetric (symmetric) states in the NS-NS (RR) order two twisted sector. The closed string twisted sector for a $\mathbf{C}^{2} / \mathbf{Z}_{\mathbf{N}}$ point contains $P-1$ hyper- and $P$ tensor multiplets of $D=6 \mathcal{N}=1$ supersymmetry. When open string sectors are present, this projection corresponds to a choice of Chan-Paton matrices with vector structure, of the form (A.5), for D5-, D9-branes, and orientifold actions (A.6) for D5-branes and (A.7) for D9-branes. The closed string twisted tadpoles at each $\mathbf{C}^{2} / \mathbf{Z}_{\mathbf{N}}$ orientifold point are

$$
\mathcal{T}_{\theta^{k}}=-32 \delta_{k, 1 \bmod 2} \quad k=1, \ldots, N-1
$$

In contrast with the previous case, the case $N=2$ leads to non-zero $\theta$-twisted tadpoles, which we may cancel by introducing e.g. D5- and $\overline{\mathrm{D} 5}$-branes.

Let us discuss the construction of the $\mathbf{B}$-type orientifold of $\mathbf{T}^{\mathbf{4}} / \mathbf{Z}_{2}$. The untwisted closed string sector is as in previous subsection, giving the $D=6, \mathcal{N}=1$ supergravity multiplet, the dilaton tensor multiplet, and two hyper- and two tensor multiplets. In the closed string twisted sector, instead, each $\alpha$-invariant $\mathbf{Z}_{\mathbf{2}}$ point give one tensor multiplet. The remaining twelve $\mathbf{Z}_{\mathbf{2}}$ points still contribute a total of six hyper- and six tensor multiplets. We achieve cancellation of twisted RR tadpoles by introducing an equal number of branes and antibranes, distributed among the four $\Omega^{\prime} \alpha$-invariant fixed points $P$, and cancelling the crosscap tadpole (2.2) for $k=1$

$$
4\left(\operatorname{Tr} \gamma_{\theta, 5, P}-\operatorname{Tr} \gamma_{\theta, \overline{5}, P}\right)-32=0
$$

The condition can be satisfied in different ways. A simple possibility is to locate one set of D5-branes with $\gamma_{\alpha^{2}, 5}=\mathbf{1}_{8}$ at two of the fixed points of $\alpha$, and two $\overline{\mathrm{D} 5}$-branes with $\gamma_{\alpha^{2}, \overline{5}}=-\mathbf{1}_{8}$ at the remaining two. The remaining $\alpha^{2}$-fixed points are not fixed under $\Omega^{\prime} \alpha$, and generate no crosscap tadpoles, and do not require any branes. The complete spectrum for this model is provided in Table 2. It is easy to see that all irreducible sixdimensional gravitational and gauge anomalies [28] cancel. The remaining factorized anomaly is cancelled by the generalized Green-Schwarz mechanism [29].

Another simple choice of open string sector satisfying all constraints is to locate, at each of the four $\alpha$-fixed points, a set of four D5-branes and four $\overline{\mathrm{D} 5}$-branes, with

$$
\gamma_{\alpha^{2}, 5}=\mathbf{1}_{4} \quad ; \quad \gamma_{\alpha^{2}, 5}=-\mathbf{1}_{4}
$$

which leads to local cancellation of their untwisted RR charge. The corresponding open string spectrum at each such point is

$$
\text { Gauge Bosons } \quad S O(4)_{D 5} \times U S p(4)_{\overline{D 5}}
$$




$$
\begin{array}{rc}
\text { Fermion }_{+} & (6 ; 1)+(1 ; 5+1)+(4 ; 4) \\
\text { Cmplx. Scalars } & (4 ; 4)
\end{array}
$$

and leads to a different anomaly-free model. Notice that, despite the branes and antibranes sit at the same fixed point, the tachyon in the $5 \overline{5}, \overline{5} 5$ groundstates is projected out by the orbifold projection. This reflects the fact that the branes and antibranes carry different twisted charges and cannot annihilate into the vacuum.

\begin{tabular}{|c|c||c|}
\hline Closed & Untwisted & $\mathcal{N}=1$ SUGRA $+3 \mathbf{T}+2 \mathbf{H}$ \\
\hline & Twisted & $10 \mathbf{T}+6 \mathbf{H}$ \\
\hline \hline Open & $\mathbf{5 5}$ & $\mathcal{N}=1$ Vector of $S O(8)^{2}$ \\
\hline & $\overline{\mathbf{5}} \overline{5}$ & ${\text { Gauge Bosons of } U S p(8)^{2}}^{2}(\mathbf{2 7}+\mathbf{1}, \mathbf{1})+(\mathbf{1}, \mathbf{2 7}+\mathbf{1})$ \\
\hline
\end{tabular}

Table 2: Spectrum of the $\Omega^{\prime} \alpha$ orientifold of $\mathbf{T}^{4} / \mathbf{Z}_{\mathbf{2}}$ with $\mathbf{B}$-type projection.

The above two solutions can actually be continuously connected, by nucleating of brane-antibrane pairs in the bulk, distributing them among the fixed points, and annihilating fractional branes (notice however that this process is suppressed by energetic barriers, hence does not alter the stability of the configuration). In fact, it is possible to construct a large set of models connected in this way. The general tachyon-free configuration is obtained by placing D5-branes and $\overline{\mathrm{D} 5}$-branes at the points $P_{r}, r=1, \ldots, 4$, with

$$
\gamma_{\theta, 5, P_{r}}=\mathbf{1}_{N_{r}} \quad ; \quad \gamma_{\theta, \overline{5}, P_{r}}=-\mathbf{1}_{8-N_{r}}
$$

Twisted tadpoles are cancelled by the above choice, and untwisted disk tadpoles of branes and antibranes cancel each other if $\sum_{r=1}^{4} N_{r}=16$. Also, the tachyonic ground states in $5 \overline{5}$ and $\overline{5} 5$ sector are projected out. This class of models contains as particular cases the possibilities considered above. Concerning which configuration is energetically favoured and provides the global minimum of the model (keeping geometry fixed), the analysis of forces between branes and antibranes at orbifold singularities in [30] seems to suggest the preferred configurations should not contains branes and antibranes at the same fixed point.

The models we have constructed are absolutely stable against decay to supersymmetric configurations, since no supersymmetric configuration of branes can satisfy the 
tadpole cancellation conditions. The models, however, can suffer diverse phase transitions as geometry is varied. In Section 6 we discuss the phase diagram of this model for the distribution of branes leading to the spectrum in Table 2. The reader interested only in this analysis is encouraged to skip Sections 3, 4 and 5.

As mentioned above, there are no other compact six-dimensional examples using the orientifold group structure mentioned above (in Section 4 we consider further sixdimensional examples, based on a different orientifold group). In the non-compact setup, however, the orientifold group structure of [27] defines a set of orientifold planes (in the sense that are fixed under an orientation-reversing action), preserving half of the supersymmetries, and which have no charge under untwisted RR fields, and twisted charges (2.1) and (2.2) for the $\mathbf{A}$ - and $\mathbf{B}$-type projections, respectively. Even though it would be interesting to explore the properties of these 'twisted orientifold planes' in more detail, e.g. in the boundary state formalism, we leave the discussion here and turn to the four-dimensional case.

\section{Four-dimensional examples}

It is straightforward to consider the construction of four-dimensional models analogous to the six-dimensional examples above. Namely we consider a crystallographic $\mathbf{Z}_{\mathbf{2 N}}$ action $\alpha$, with a vector of twist eigenvalues $\tilde{v}=\frac{1}{2 N}\left(a_{1}, a_{2}, a_{3}\right)$. We center on models preserving supersymmetry in the closed string sector, and so impose $\sum_{i} a_{i}=0(\bmod 2 N)$. Without loss of generality we may take $a_{1}, a_{2}$ odd and $a_{3}$ even. We consider type IIB theory on the orbifold $\mathbf{T}^{\mathbf{6}} / \mathbf{Z}_{\mathbf{N}}$, where $\mathbf{Z}_{\mathbf{N}}$ is generated by $\theta=\alpha^{2}$, with twist vector $v=2 \tilde{v}=\frac{1}{N}\left(a_{1}, a_{2}, a_{3}\right)$. Our models are obtained by modding out by the orientifold action $\Omega^{\prime} \alpha$, with $\Omega^{\prime}=\Omega R_{1} R_{2} R_{3}(-1)^{F_{L}}$, where $R_{i}: z_{i} \rightarrow-z_{i}$, and $F_{L}$ is left-handed world-sheet fermion number. The D-branes surviving the $\Omega^{\prime} \alpha$ projection, and which we may use to cancel the tadpoles are D3-branes (at a point in the internal space) and D7 $i$-branes (transverse to the $i^{\text {th }}$ complex plane), and the corresponding antibranes. Use of other orientifold projection, like $\Omega \alpha$ would lead to T-dual version of our models.

Crystallographic actions on $\mathbf{T}^{\mathbf{6}}$ compatible with unbroken supersymmetry have been classified in the early orbifold literature [31], and we show the possible choices for $\alpha$ and $\theta=\alpha^{2}$ in Table 3. Notice that, since we are interested in models without untwisted tadpoles, we have already restricted to even $N, N=2 P$.

\footnotetext{
${ }^{3}$ Considering $\sum_{i} a_{i}=N \bmod N$ also leads to $\mathcal{N}=1$ supersymmetric models. It is possible to show, however, that they are equivalent to the ones we consider.

${ }^{4}$ Extension to orbifolds $\mathbf{T}^{\mathbf{6}} /\left(\mathbf{Z}_{\mathbf{N}} \times \mathbf{Z}_{\mathbf{M}}\right)$, not considered in the present paper, is straightforward.
} 


\begin{tabular}{|c||c||c|}
\hline$\Gamma$ & $\Omega^{\prime} \alpha$ & $\theta$ \\
\hline $\mathbf{Z}_{\mathbf{2}}$ & $\tilde{v}=\frac{1}{4}(1,1,-2)$ & $v=\frac{1}{2}(1,1,-2)$ \\
\hline $\mathbf{Z}_{\mathbf{4}}$ & $\tilde{v}=\frac{1}{8}(1,3,-4)$ & $v=\frac{1}{4}(1,3,-4)$ \\
\hline $\mathbf{Z}_{\mathbf{4}}$ & $\tilde{v}=\frac{1}{8}(1,-3,2)$ & $v=\frac{1}{4}(1,-3,2)$ \\
\hline $\mathbf{Z}_{\mathbf{6}}$ & $\tilde{v}=\frac{1}{12}(1,-5,4)$ & $v=\frac{1}{6}(1,-5,4)$ \\
\hline $\mathbf{Z}_{\mathbf{6}}$ & $\tilde{v}=\frac{1}{12}(1,5,-6)$ & $v=\frac{1}{6}(1,5,-6)$ \\
\hline
\end{tabular}

Table 3: Twist eigenvalues for the $\Omega^{\prime} \alpha$ orientifolds of $\mathbf{T}^{\mathbf{6}} / \mathbf{Z}_{\mathbf{N}}$ orbifolds.

As in the six-dimensional case, for even $N$ there exist two consistent orientifold projections, which we denote of $\mathbf{A}$ - and $\mathbf{B}$-type as well, differing in their action on the order two twisted sector. The A-type projection chooses left-right symmetric (antisymmetric) states in the twisted NS-NS (RR) sector, while the B-type projection does the opposite. At the level of the massless closed string spectrum this amounts to choosing either a $D=4, \mathcal{N}=1$ chiral or a linear multiplet in the corresponding sector. Given chiral-linear duality in four dimensions, both projections lead to a similar closed string spectrum (even though in some instances, like cancellation of $U(1)$ anomalies [32, it is important to distinguish chiral and linear multiplets [33, 34). The closed string spectra for the models in Table 3 are provided in Table 4 . Notice the unusual appearance of vector multiplets in the untwisted sector. In geometrical terms, they arise from the reduction of the type IIB self-dual 4 -form, odd under $\Omega$, on $(2,1)$-forms odd under the geometric action in $\Omega^{\prime} \alpha$.

However, the two projections differ markedly in the twisted tadpoles they generate, and therefore in the required open string spectra, as we discuss in the following.

\subsection{Models with A-type projection}

The computation of the twisted crosscap tadpoles can be carried out by following standard techniques. In the appendix, we compute these twisted charges by merely adapting to our orientifold action the computations for four-dimensional models in the Appendix of [9]. We would like to point out that, although our interest in the present paper is centered on compact models, the calculation is valid for the general non-compact case of $\Omega^{\prime} \alpha$ orientifolds of $\mathbf{C}^{\mathbf{3}} / \mathbf{Z}_{\mathbf{N}}$, where one drops the crystallographic condition on the twists. Hence, such models can be used to define a large family of 'twisted orientifold planes', uncharged with respect to untwisted RR fields, but with 


\begin{tabular}{|c|c||c|c|}
\hline $\mathbf{T}^{\mathbf{6}} / \mathbf{Z}_{\mathbf{N}}$ & $\Omega^{\prime} \alpha$ twist & Untwisted & Twisted \\
\hline $\mathbf{Z}_{\mathbf{2}}$ & $\tilde{v}=\frac{1}{4}(1,1,-2)$ & $6 \mathbf{V}+8 \mathbf{C h}$ & $6 \mathbf{V}+26 \mathbf{C h}$ \\
\hline $\mathbf{Z}_{\mathbf{4}}$ & $\tilde{v}=\frac{1}{8}(1,3,-4)$ & $4 \mathbf{V}+6 \mathbf{C h}$ & $8 \mathbf{V}+28 \mathbf{C h}$ \\
\hline $\mathbf{Z}_{\mathbf{4}}$ & $\tilde{v}=\frac{1}{8}(1,-3,2)$ & $1 \mathbf{V}+5 \mathbf{C h}$ & $3 \mathbf{V}+29 \mathbf{C h}$ \\
\hline $\mathbf{Z}_{\mathbf{6}}$ & $\tilde{v}=\frac{1}{12}(1,-5,4)$ & $5 \mathbf{C h}$ & $2 \mathbf{V}+27 \mathbf{C h}$ \\
\hline $\mathbf{Z}_{\mathbf{6}}$ & $\tilde{v}=\frac{1}{12}(1,5,-6)$ & $4 \mathbf{V}+6 \mathbf{C h}$ & $8 \mathbf{V}+28 \mathbf{C h}$ \\
\hline
\end{tabular}

Table 4: Closed string spectra for the $\Omega^{\prime} \alpha$ orientifolds of $\mathbf{T}^{\mathbf{6}} / \mathbf{Z}_{\mathbf{N}}$ orbifolds. We have not included the universally present $\mathcal{N}=1$ sugra multiplet and dilaton linear multiplet.

non-zero twisted charges. The crosscap tadpoles for this type of orientifold models are

$$
\mathcal{T}_{\theta^{k}}=-16 \delta_{k, 1 \bmod 2}\left[\sin \left(\pi k v_{1}\right)+\sin \left(\pi k v_{2}\right)\right] \quad, \quad k=1, \ldots, N-1
$$

Our twisted charges are normalized in units such that the $\theta^{k}$-twisted charges of D3and $\mathrm{D} 7_{i}$-branes are $8 \prod_{j=1}^{3} \sin \left(\pi k v_{j}\right) \operatorname{Tr} \gamma_{\theta^{k}, 3}$ and $2 \sin \left(\pi k v_{i}\right) \operatorname{Tr} \gamma_{\theta^{k}, 7_{i}}$, respectively.

In the four-dimensional case, twisted tadpoles may have non-trivial dependence with the compactification volumes, denoted $V_{i}$ for the $i^{\text {th }}$ complex plane (we also introduce a regularized volume $V_{4}$ for four-dimensional spacetime). Concretely, a crosscap twisted by $\Omega^{\prime} \alpha$ produces a tadpole proportional to $\sqrt{V_{i}}$ if $\alpha^{2 k+1}$ reflects the $i^{\text {th }}$ plane, and proportional to $1 / \sqrt{V_{i}}$ if it leaves $z_{i}$ invariant. A disk twisted by $\theta^{k}$ produces a tadpole proportional to $\sqrt{V_{i}}$ if $z_{i}$ is a Neumann direction left invariant by $\theta^{k}$, and proportional to $1 / \sqrt{V_{i}}$ if it $z_{i}$ is a Dirichlet direction left invariant by $\theta^{k}$. Any tadpole is proportional to $\sqrt{V_{4}}$. These dependences are implicitly taken into account in all tadpole cancellations in this paper.

In general, cancellation of twisted tadpoles will require the introduction of open string sectors. The A-type projection corresponds to a choice of Chan-Paton matrices (A.1) and (A.2) for D3-branes, (A.1) and (A.3) for D7 3 -branes, and (A.5) and (A.7) for both $\mathrm{D} 7_{1}$ - and $\mathrm{D} 7_{2}$-branes. @.

There are several compact models which, however, do not lead to twisted tadpoles. This is the case for the orientifolds with $\Omega^{\prime} \alpha$ twists given by $\tilde{v}=\frac{1}{8}(1,-3,2)$ and $\tilde{v}=\frac{1}{12}(1,-5,4)$. These models do not require open string sectors, and define consistent

\footnotetext{
${ }^{5}$ This flip between the vector structure of different branes can be shown as in [35]. Note the ambiguity in labeling the two projections in the closed sector as with and without vector structure, and the advantage of our labeling as $\mathbf{A}$ - and $\mathbf{B}$-type.
} 
unoriented closed string theories. The corresponding closed string spectra can be read from the relevant rows in Table 1 .

The remaining cases correspond to orientifolds with non-zero twisted tadpoles, which we may try to cancel by the introduction of e.g. a suitable set of branes and antibranes. We describe them in the following,

- The $\tilde{v}=\frac{1}{4}(1,1,-2)$ model

The contribution from the $\Omega^{\prime} \alpha, \Omega^{\prime} \alpha^{3}$ crosscaps to the $\theta$-twisted tadpole (3.1) is proportional to $\sqrt{V_{4} V_{3}}$. This cannot be cancelled by using D3- or D73-branes, but it can be cancelled by $\mathrm{D} 7_{1^{-}}$or $\mathrm{D} 7_{2}$-branes. Here we describe a particular solution to the tadpole conditions using an equal number of $\mathrm{D} 7_{1^{-}}$and $\overline{\mathrm{D}}_{1}$-branes. There are sixteen fixed points of $\Omega^{\prime} \alpha$, with coordinates $\left(z_{1}, z_{2}, z_{3}\right)$ with $z_{1}=0,(1+i) / 2, z_{2}=0,(1+i) / 2$, $z_{3}=0,1 / 2, i / 2,(1+i) / 2$. At each of these points, indexed by $P$, we need

$$
\operatorname{Tr} \gamma_{\theta, 7_{1}, P}-\operatorname{Tr} \gamma_{\theta, \overline{7}_{1}, P}-16=0
$$

There are also sixteen fixed tori of $\theta$, out of which four (with coordinates $\left(z_{1}, z_{2}\right)$, with $\left.z_{1}=0,(1+i) / 2, z_{2}=0,(1+i) / 2\right)$ are fixed under $\Omega^{\prime} \alpha$, and twelve are not. At the latter, indexed by $Q, \theta$-twisted crosscap tadpoles are not generated, and hence the corresponding twisted charges of the $\mathrm{D} 7_{1}$ - and $\overline{\mathrm{D}}_{1}$-branes should be zero.

$$
\operatorname{Tr} \gamma_{\theta, 7_{1}, Q}-\operatorname{Tr} \gamma_{\theta, \overline{7}_{1}, Q}=0
$$

We consider the following solution. We locate $16 \mathrm{D} 7_{1}$-branes at $z_{1}=0$ in the first plane, and $16 \overline{\mathrm{D}}_{1}$-branes at $z_{1}=(1+i) / 2$, with

$$
\gamma_{\theta, 7_{1}, 0}=\mathbf{1}_{16} \quad, \quad \gamma_{\theta, \overline{7}_{1},(1+i) / 2}=-\mathbf{1}_{16}
$$

which satisfy the $\theta$-tadpole condition (3.2) at the points $P$. In order to avoid the contribution of twisted charges to the points $Q$ which they cross, we introduce Wilson lines along the second complex plane

$$
\gamma_{W_{2}, 7_{1}, 0}=\operatorname{diag}\left(\mathbf{1}_{8},-\mathbf{1}_{8}\right) \quad, \quad \gamma_{W_{2}, \overline{7}_{1},(1+i) / 2}=\operatorname{diag}\left(\mathbf{1}_{8},-\mathbf{1}_{8}\right)
$$

This completes the construction of the model. The resulting open string spectrum is given in Table 5 .

- The $\tilde{v}=\frac{1}{8}(1,3,-4)$ model

In this model, the crosscaps associated to $\Omega^{\prime} \alpha$ and $\Omega^{\prime} \alpha^{3}$ produce a contribution to the $\theta-, \theta^{3}$-twisted tadpoles proportional to $\sqrt{V_{4} V_{3}}$. As in the previous model, they may be cancelled by the introduction of $\mathrm{D} 7_{1^{-}}$or $\mathrm{D} 7_{2}$-branes. In the following we construct a particular solution using an equal number of $\mathrm{D} 7_{1^{-}}$and $\overline{\mathrm{D}}_{1^{-}}$-branes. 


\begin{tabular}{|c|c|}
\hline $7_{1} 7_{1}$ & $\mathcal{N}=1$ Vectors of $U S p(8)^{2}$ \\
\hline & $\mathcal{N}=1$ Chiral $(\mathbf{3 6}, \mathbf{1})+(\mathbf{1}, \mathbf{3 6})$ \\
\hline \multirow[t]{4}{*}{$\overline{\mathbf{7}}_{1} \overline{\mathbf{7}}_{1}$} & Gauge Bosons of $S O(8)^{2}$ \\
\hline & Fermion $_{+}(\mathbf{3 5}+\mathbf{1}, \mathbf{1})+(\mathbf{1}, \mathbf{3 5}+\mathbf{1})$ \\
\hline & Cmplx. Scalars $(\mathbf{2 8 , 1})+(\mathbf{1}, \mathbf{2 8})$ \\
\hline & Fermion $_{-}(\mathbf{3 5}+\mathbf{1}, \mathbf{1})+(\mathbf{1}, \mathbf{3 5}+\mathbf{1})$ \\
\hline
\end{tabular}

Table 5: Open string spectrum of the $\Omega^{\prime} \alpha$ orientifold $\tilde{v}=\frac{1}{4}(1,1,-2)$ of $\mathbf{T}^{\mathbf{4}} / \mathbf{Z}_{\mathbf{2}}$ with A-type projection.

The model contains eight $\Omega^{\prime} \alpha$-fixed points, at the locations denoted by $\left(P_{1}, z_{3}\right)$, $\left(P_{2}, z_{3}\right)$, where $z_{3}=0,1 / 2, i / 1,(1+i) / 2$, and $P_{j}$ denotes the location of the fixed points in the first two complex planes, whose explicit form is not necessary here. At each of these eight points, indexed by $P$, we need

$$
\operatorname{Tr} \gamma_{\theta, 7_{1}, P}-\operatorname{Tr} \gamma_{\theta, \overline{7}_{1}, P}-16=0
$$

There are also additional fixed points of $\theta$ which are not fixed under $\Omega^{\prime} \alpha$, and do not generate crosscap tadpoles. At these points, indexed by $Q$, we need

$$
\operatorname{Tr} \gamma_{\theta, 7_{1}, Q}-\operatorname{Tr} \gamma_{\theta, \overline{7}_{1}, Q}=0
$$

Finally, the closed string sector does not generate $\theta^{2}$-twisted tadpoles, hence the disk contributions should also vanish. At all points of $P$ or $Q$ type we need

$$
\operatorname{Tr} \gamma_{\theta^{2}, 7_{1}}-\operatorname{Tr} \gamma_{\theta^{2}, \overline{7}_{1}}=0
$$

We consider the following solution. We locate $32 \mathrm{D} 7_{1}$-branes passing through $P_{1}$ and $32 \overline{\mathrm{D}}_{1}$-branes passing through $P_{2}$, with

$$
\gamma_{\theta, 7_{1}, P_{1}}=\operatorname{diag}\left(\mathbf{1}_{16}, e^{2 \pi i \frac{1}{4}} \mathbf{1}_{8}, e^{2 \pi i \frac{3}{4}} \mathbf{1}_{8}\right) \quad ; \quad \gamma_{\theta, \overline{7}_{1}, P_{2}}=\operatorname{diag}\left(e^{2 \pi i \frac{1}{4}} \mathbf{1}_{8}, e^{2 \pi i \frac{2}{4}} \mathbf{1}_{16}, e^{2 \pi i \frac{3}{4}} \mathbf{1}_{8}\right)(3.9)
$$

Notice that fixed points of $\theta$ not fixed under $\Omega^{\prime} \alpha$ (i.e. of type $Q$ ) remain empty and satisfy (3.7) automatically. The resulting spectrum is shown in Table 6 .

- The $\tilde{v}=\frac{1}{12}(1,5,-6)$ model

In this model there are four $\Omega^{\prime} \alpha$-fixed points, which we denote by $\left(0, z_{3}\right)$, where 0 represents the origin in the first two complex planes, and $z_{3}=0,1 / 2, i / 1,(1+i) / 2$ gives their location in the third. At these points $P$ there are crosscap tadpoles for $\theta$ - and 


\begin{tabular}{|c||c|}
\hline $\mathbf{7}_{1} \mathbf{7}_{1}$ & $\mathcal{N}=1$ Vectors of $U S p(16) \times U(8)$ \\
\hline & $\mathcal{N}=1$ Chiral $(\mathbf{1 6}, \overline{\mathbf{8}})+(\mathbf{1 6}, \mathbf{8})+(\mathbf{1 3 6}, \mathbf{1})+(\mathbf{1}, \mathrm{Adj})$ \\
\hline$\overline{\mathbf{7}}_{1} \overline{\mathbf{7}}_{1}$ & Gauge Bosons of $U(8) \times S O(16)$ \\
\hline & Fermion $_{+}(\mathbf{A d j}, \mathbf{1})+(\mathbf{1}, \mathbf{1 3 5}+\mathbf{1})$ \\
\hline & Cmplx. Scalars $(\mathbf{8}, \mathbf{1 6})+(\overline{\mathbf{8}}, \mathbf{1 6})+(\mathrm{Adj}, \mathbf{1})+(\mathbf{1}, \mathbf{1 2 0})$ \\
\hline & Fermion $_{-}(\mathbf{8}, \mathbf{1 6})+(\overline{\mathbf{8}}, \mathbf{1 6})+(\mathrm{Adj}, \mathbf{1})+(\mathbf{1}, \mathbf{1 3 5}+\mathbf{1})$ \\
\hline
\end{tabular}

Table 6: Open string spectrum of the $\Omega^{\prime} \alpha$ orientifold $\tilde{v}=\frac{1}{8}(1,3,-4)$ of $\mathbf{T}^{\mathbf{4}} / \mathbf{Z}_{\mathbf{4}}$ with A-type projection.

$\theta^{3}$-twisted fields, proportional to the volume factor $\sqrt{V_{4} V_{3}}$. In order to cancel them we introduce an equal number of $\mathrm{D} 7_{1^{-}}$and $\overline{\mathrm{D}}_{1}$-branes, whose Chan-Paton matrices are then constrained by

$$
\begin{array}{r}
\operatorname{Tr} \gamma_{\theta, 7_{1}, P}-\operatorname{Tr} \gamma_{\theta, \overline{7}_{1}, P}-16=0 \\
\operatorname{Tr} \gamma_{\theta^{3}, 7_{1}, P}-\operatorname{Tr} \gamma_{\theta^{3}, \overline{7}_{1}, P}-16=0
\end{array}
$$

In this model there are no additional $\theta$-fixed points. The only remaining constraint is the cancellation of $\theta^{2}$-twisted disk tadpoles (since there are no corresponding crosscap contribution). We have

$$
\operatorname{Tr} \gamma_{\theta^{2}, 7_{1}, P}-\operatorname{Tr} \gamma_{\theta^{2}, \overline{7}_{1}, P}=0
$$

The simplest solution is to locate $\mathrm{D} 7_{1^{-}}$and $\overline{\mathrm{D}}_{1}$-branes passing through the origin, and with

$$
\gamma_{\theta, 7_{1}}=\mathbf{1}_{8} \quad ; \quad \gamma_{\theta, \overline{7}_{1}}=-\mathbf{1}_{8}
$$

Notice that since the Chan-Paton matrices above have no common eigenvalues, the tachyonic $7_{1} \overline{7}_{1}, \overline{7}_{1} 7_{1}$ groundstates are projected out. The resulting spectrum is shown in Table 7

\subsection{Models with B-type projection}

The twisted crosscap tadpoles for a $\Omega^{\prime} \alpha$ orientifold of $\mathbf{C}^{\mathbf{3}} / \mathbf{Z}_{\mathbf{N}}$ with the $\mathbf{B}$-type projection are computed in the appendix, leading to the result

$$
\mathcal{T}_{\theta^{k}}=+16 \sin \left(\pi k v_{3}\right) \delta_{k, 1 \bmod 2}, \quad k=1, \ldots, N-1
$$




\begin{tabular}{|c|c|}
\hline \multirow[t]{2}{*}{$7_{1} 7_{1}$} & $\mathcal{N}=1$ Vectors of $U S p(8)$ \\
\hline & $\mathcal{N}=1$ Chiral 36 \\
\hline \multirow[t]{4}{*}{$\overline{\mathbf{7}}_{1} \overline{\mathbf{7}}_{1}$} & Gauge Bosons of $S O(8)$ \\
\hline & Fermion $_{+} \mathbf{3 5}+\mathbf{1}$ \\
\hline & Cmplx. Scalars \\
\hline & 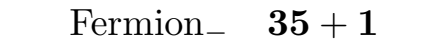 \\
\hline
\end{tabular}

Table 7: Spectrum of the $\Omega^{\prime} \alpha$ orientifold $\tilde{v}=\frac{1}{12}(1,5,-6)$ of $\mathbf{T}^{\mathbf{4}} / \mathbf{Z}_{\mathbf{6}}$ with $\mathbf{A}$-type projection.

In some of the models in Table 3 all twisted tadpoles vanish. Therefore they do not require open string sector, and define consistent unoriented closed string theories in four dimensions. This is the case for the models with $\Omega^{\prime} \alpha$ twists $\tilde{v}=\frac{1}{4}(1,1,-2)$, $\tilde{v}=\frac{1}{8}(1,3,-4)$, and $\tilde{v}=\frac{1}{12}(1,5,-6)$. The corresponding closed string spectra can be read from the relevant rows in Table 4 .

The remaining cases correspond to orientifolds with non-zero twisted tadpoles, which may be cancelled by the introduction of e.g. a suitable set of branes and antibranes. In the following we describe these models.

- The $\tilde{v}=\frac{1}{8}(1,-5,4)$ model

In this $\Omega^{\prime} \alpha$ orientifold of $\mathbf{T}^{\mathbf{6}} / \mathbf{Z}_{\mathbf{8}}$, the only twisted tadpoles in the compact model reside at the four $\alpha$ fixed points, indexed by $P$. We consider a model in which they are cancelled by the introduction of an equal number of D3- and $\overline{\mathrm{D} 3}$-branes. The conditions of cancellation of the twisted RR tadpoles (3.13) for $k=1 \mathrm{read}$

$$
\operatorname{Tr} \gamma_{\theta, 3, P}-\operatorname{Tr} \gamma_{\theta, \overline{3}, P}=4
$$

For $k=2$ the twisted crosscap tadpoles (3.13) vanishes. Since $\theta^{2}$ has a fixed plane, all D3- $\overline{\mathrm{D} 3}$-branes at points $P$ are sources for the same $\theta^{2}$-twisted field. The condition of cancellation of charge then reads

$$
\sum_{P}\left(\operatorname{Tr} \gamma_{\theta^{2}, 3, P}-\operatorname{Tr} \gamma_{\theta^{2}, \overline{3}, P}\right)=0
$$

The simplest solution to these conditions is to locate, at two of the $\Omega^{\prime} \alpha$-fixed points, four D3-branes, and four $\overline{\mathrm{D} 3}$-branes at each of the remaining two, with

$$
\gamma_{\theta, 3}=\mathbf{1}_{4} \quad, \quad \gamma_{\theta, \overline{3}}=-\mathbf{1}_{4}
$$




\begin{tabular}{|l||c|}
\hline $\mathbf{3 3}$ & $\mathcal{N}=1$ Vectors of $S O(4)^{2}$ \\
\hline$\overline{\mathbf{3}} \overline{\mathbf{3}}$ & ${\text { Gauge Bosons of } U S p(4)^{2}}^{2}$ \\
\hline & Fermion $_{+} \quad(\mathbf{5}+\mathbf{1}, \mathbf{1})+(\mathbf{1}, \mathbf{5}+\mathbf{1})$ \\
\hline
\end{tabular}

Table 8: Open string spectrum of the $\Omega^{\prime} \alpha$ orientifold (with twist $\tilde{v}=\frac{1}{8}(1,-3,2)$ ) of $\mathbf{T}^{\mathbf{6}} / \mathbf{Z}_{\mathbf{4}}$ with $\mathbf{B}$-type projection.

The resulting spectrum is given in Table 8

- The $\tilde{v}=\frac{1}{12}(1,-5,4)$ model

We conclude with the discussion of this $\Omega^{\prime} \alpha$ orientifold of $\mathbf{T}^{\mathbf{6}} / \mathbf{Z}_{\mathbf{6}}$, and by showing there is no configuration of branes and antibranes leading cancellation of all twisted tadpoles.

The argument goes as follows. First notice that we can treat branes and antibranes in a unified manner by using only branes but formally allowing the multiplicities of their Chan-Paton eigenvalues to take negative values. The $\Omega^{\prime} \alpha$ crosscaps generate a $\theta$-twisted tadpole at the three $\alpha$-fixed points, which sit at the origin in the first two planes, and at three points, labeled by $n=0, \pm 1$ in the third. We introduce a general configuration of branes, passing through these points. We include $\mathrm{D} 7_{1}$ - and $\mathrm{D} 72_{2}$-branes passing through the origin in the first two planes, and with arbitrary order three Wilson lines $\gamma_{W_{3}, 7_{1}}, \gamma_{W_{3}, 7_{2}}$ in the third. We also consider $\mathrm{D} 7_{3}$-branes sitting at the three different locations in the third plane, and D3-branes, at the origin in the first two planes, and in the three locations in the third. The $\theta$-twisted tadpole equation reads

$$
\frac{1}{\sqrt{3}} \operatorname{Tr}\left(\gamma_{\theta, 7_{1}} \gamma_{W_{3}, 7_{1}}^{n}\right)-\frac{1}{\sqrt{3}} \operatorname{Tr}\left(\gamma_{\theta, 7_{2}} \gamma_{W_{3}, 7_{2}}^{n}\right)+\operatorname{Tr} \gamma_{\theta, 7_{3}, n}-\operatorname{Tr} \gamma_{\theta, 3, n}+8=0
$$

The crosscap twisted by $\Omega^{\prime} \alpha^{3}$ generates a tadpole in the $\theta^{3}$-twisted sector, proportional to the volume factor $\sqrt{V_{4} / V_{3}}$. This $\theta^{3}$-tadpole also receives contributions from disks twisted by $\theta^{3}$ associated with $\mathrm{D} 3$ - and $\mathrm{D} 7_{3}$-brane, regardless of their location in the third plane. There is no contribution with this volume dependence from $\mathrm{D} 7_{1}$ - or $\mathrm{D} 7_{2}$-branes, hence the $\theta^{3}$ tadpole condition reads

$$
\sum_{n}\left(\operatorname{Tr} \gamma_{\theta^{3}, 7_{3}, n}-4 \operatorname{Tr} \gamma_{\theta^{3}, 3, n}\right)+8=0
$$

We now show that (3.17) and (3.18) are incompatible. It is enough to consider the equations mod 3. Let us sum (3.17) over $n$, and take the resulting equation mod 3 . 
We obtain

$$
\begin{aligned}
& \frac{1}{\sqrt{3}} \operatorname{Tr} {\left[\gamma_{\theta, 7_{1}}\left(\sum_{n=0, \pm 1} \gamma_{W_{3}, 7_{1}}^{n}\right)\right]-\frac{1}{\sqrt{3}} \operatorname{Tr}\left[\gamma_{\theta, 7_{2}}\left(\sum_{n=0, \pm 1} \gamma_{W_{3}, 7_{2}}^{n}\right)\right]+} \\
&+\sum_{n=0, \pm 1} \operatorname{Tr} \gamma_{\theta, 7_{3}, n}-\sum_{n=0, \pm 1} \operatorname{Tr} \gamma_{\theta, 3, n}=0 \bmod 3
\end{aligned}
$$

Expressing the D3- Chan-Paton traces in terms of the eigenvalue multiplicities from (A.5), we have

$$
\operatorname{Tr} \gamma_{\theta, 3, n}=n_{0}+n_{1}-n_{2}-n_{3}=n_{0}-2 n_{1}+2 n_{2}-n_{3} \bmod 3=\operatorname{Tr} \gamma_{\theta^{3}, 3, n} \bmod 3
$$

and analogously for $\mathrm{D}_{3}$-branes. Also, since the Wilson line $W_{3}$ is of order three, the eigenvalues in $\gamma_{W_{3}, 7_{1}}$ are $1, e^{ \pm 2 \pi i / 3}$, and we can show $\sum_{n} \gamma_{W_{3}, 7_{1}}^{n}=0 \bmod 3$. Finally, it is important to notice that the $\mathrm{D} 7_{1^{-}}$and $\mathrm{D} 7_{2}$-brane Chan-Paton matrices are of the form (A.1), without vector structure, hence their contributions $\operatorname{tr} \gamma_{\theta}$ in (3.19) are of the form an integer times $\sqrt{3}$, this latter factor cancelling the coefficient $1 / \sqrt{3}$.

Using these properties in (3.19), we get

$$
\sum_{n}\left(\operatorname{Tr} \gamma_{\theta^{3}, 7_{3}, n}-4 \operatorname{Tr} \gamma_{\theta^{3}, 3, n}\right)=0 \bmod 3
$$

in contradiction with (3.18). Notice that there may be additional contribution to (3.18) from additional D3- or $\mathrm{D} 73_{3}$-branes sitting at the origin in the first two planes, and at arbitrary points (not $\theta$-fixed) in the third. However, these objects necessarily come in three identical copies, permuted by the action of $\theta$, and their contribution is zero mod 3, and does not change the argument above. We have not found any additional contributions which could help in satisfying the tadpole conditions, and so have not succeeded in constructing a consistent version of this orientifold model.

It is interesting to rephrase the problem we have encountered in a more abstract language. Given an orientifold model, one can envision the space of all possible (untwisted and twisted) charges under its RR fields, which we loosely refer to as the charge lattice (even though some charges may not be $\mathbf{Z}$-valued). The charges of the orientifolds in the model define a vector in that space, and the problem of cancelling the RR tadpoles corresponds to constructing a state of the theory, typically a configuration of branes, with precisely that charge vector. Clearly, a problem arises if the charge vector defined by the crosscap charges corresponds to an empty site in the charge lattice, i.e. a set of charges for which no state in the theory exists.

This situation is precisely what we have found in the above model. We have shown that for a very general kind of brane configurations the set of states reproducing the correct $\theta$-twisted charges fills only a subset of all possible $\theta^{3}$-twisted charges, namely zero mod 3. The crosscap contribution however, corresponds to a $\theta^{3}$-twisted charge 
equal to -2 , which is an empty site in the sense explained above. Whether other kinds of branes can fill the empty site, and provide a consistent completion of the model remains an open question.

We conclude by pointing out that other orientifold models have been previously claimed to be inconsistent due to uncancelable tadpoles. In particular, the $\Omega$ orientifold of $\mathbf{T}^{\mathbf{6}} / \mathbf{Z}_{\mathbf{4}}$ with $\mathbf{A}$-type projection [9] was shown to contain a twisted tadpole with an unusual volume dependence, and whose cancellation could not be achieved with the D-branes in the model. In Section 5 we review this problem, and find that in fact it is possible to construct a set of brane-antibrane pairs canceling precisely this problematic tadpole, and rendering the model consistent. This nicely illustrates the power of nonBPS configurations of branes in cancelling twisted charges. Our above example in this Section, though, suggests there still exist orientifolds for which tadpole cancellation cannot be achieved.

\section{Further six-dimensional models}

In this section we study a set of six-dimensional models without untwisted tadpoles, obtained by modding out type IIB theory on $\mathbf{T}^{\mathbf{4}} / \mathbf{Z}_{\mathbf{N}}$, for even $N$, by the orientifold action $\Omega \Pi$, with $\Pi:\left(z_{1}, z_{2}\right) \rightarrow\left(z_{2},-z_{1}\right)$. Notice that closure requires $N$ to be even, $N=2 P$, therefore it is possible to construct compact $\Omega \Pi$ orientifolds of the $\mathbf{Z}_{\mathbf{2}}, \mathbf{Z}_{\mathbf{4}}$ and $\mathbf{Z}_{6}$ orbifolds.

This type of orientifold projection has been studied in 36 in the non-compact context, i.e. orientifolds of $\mathbf{C}^{\mathbf{2}} / \mathbf{Z}_{\mathbf{N}}$, where the crystallographic constraint can be dropped.The closed string twisted sector, for a $\mathbf{C}^{2} / \mathbf{Z}_{\mathbf{N}}$ point gives rise to $N-1$ tensor multiplets of $D=6 \mathcal{N}=1$ supersymmetry. The closed string sector contribution to twisted tadpoles can be computed to be

$$
\mathcal{T}_{\theta^{k}}=-16 N \delta_{k, N / 2}=0
$$

in units where the twisted charges of D5- and D9-branes are $4 \sin ^{2}(\pi k / N) \operatorname{Tr} \gamma_{\theta^{k}, 5}$ and $\operatorname{Tr} \gamma_{\theta^{k}, 9}$ respectively.

Since there is always at least one non-zero twisted tadpole, the configurations requires open string sectors for consistency. We will introduce sets of D5-branes and $\overline{\mathrm{D} 5}$-branes in order to cancel twisted tadpoles (D9- and $\overline{\mathrm{D} 9}$-branes may also be easily incorporated). The general structure of their Chan-Paton matrices is

$$
\begin{aligned}
\gamma_{\theta^{k}, 5} & =\operatorname{diag}\left(\mathbf{1}_{n_{0}}, e^{2 \pi i \frac{1}{N}} \mathbf{1}_{n_{1}}, \ldots, e^{2 \pi i \frac{N-1}{N}} \mathbf{1}_{n_{N-1}}\right) \\
\gamma_{\Omega \Pi, 5} & =\operatorname{diag}\left(\mathbf{1}_{n_{0}}, \varepsilon_{n_{1}}, \ldots, \mathbf{1}_{n_{N-2}}, \varepsilon_{n_{N-1}}\right)
\end{aligned}
$$


and analogously for $\overline{\mathrm{D} 5}$-branes. The open string sector, for instance in the absence of $\overline{\mathrm{D} 5}$-branes, gives rise to the following multiplets on the D5-brane world-volume

$$
\begin{array}{cr}
\text { Vector } & S O\left(n_{0}\right) \times U S p\left(n_{1}\right) \times \ldots \times S O\left(n_{N-2}\right) \times U S p\left(n_{N-1}\right) \\
\text { Hyper } & \frac{1}{2}\left(n_{0}, n_{1}\right)+\frac{1}{2}\left(n_{1}, n_{2}\right)+\ldots+\frac{1}{2}\left(n_{N-1}, n_{0}\right)
\end{array}
$$

If instead we have only $\overline{\mathrm{D} 5}$-branes and no D5-branes, we obtain a non-supersymmetric version of the above spectrum, with the only difference that the 'gauginos' transform in the 'wrong' two-index representation of the gauge group (symmetric of $S O\left(n_{2 i}\right)$ and antisymmetric of $\left.U S p\left(n_{2 i+1}\right)\right)$.

Let us turn to the construction of compact models. The $\Omega \Pi$ orientifold of the $\mathbf{T}^{\mathbf{4}} / \mathbf{Z}_{\mathbf{2}}$ orbifold is actually equivalent to the $\Omega \alpha$ orientifold of the $\mathbf{T}^{\mathbf{4}} / \mathbf{Z}_{\mathbf{2}}$ orbifold studied in Section 2, as can be shown by diagonalizing $\Pi$ without changing the structure of the orbifold twist $\theta$. Hence it does not give rise to a new model \%. The situation is different for the $\mathbf{Z}_{\mathbf{4}}$ and $\mathbf{Z}_{\mathbf{6}}$ orbifolds, since diagonalizing $\Pi$ changes the action of $\theta$.

Let us center on the $\Omega \Pi$ orientifold of $\mathbf{T}^{\mathbf{4}} / \mathbf{Z}_{\mathbf{4}}$. In the untwisted closed string sector, we obtain the $D=6 \mathcal{N}=1$ gravity multiplet, the dilaton tensor multiplet, and two hyper- and two tensor multiplets. In the twisted sector, two of the four $\theta$-fixed points are also fixed under $\Omega \Pi$, and produce three tensor multiplets each. The remaining two points are exchanged by $\Omega \Pi$, and give a total of three hyper- and three tensor multiplets. There are also twelve fixed points of $\theta^{2}$ which are grouped in pairs by the action of $\theta$, i.e. they correspond to six $\mathbf{C}^{2} / \mathbf{Z}_{2}$ singularities in the quotient. Two of them are invariant under $\Omega \Pi$, and give one tensor multiplet each, while the remaining four produce a total of two hyper- and two tensor multiplets. In total, we have six hyper- and fourteen tensor multiplets.

Points fixed under $\Omega \Pi$ generate twisted tadpoles. In order to cancel them, we introduce e.g. 16 D5-branes at one of the points fixed under $\theta$ and $\Omega \Pi$ and $16 \overline{\mathrm{D} 5}$ branes at the other, with

$$
\gamma_{\theta, 5, P_{1}}=\operatorname{diag}\left(\mathbf{1}_{8}, e^{2 \pi i \frac{2}{4}} \mathbf{1}_{8}\right) \quad ; \quad \gamma_{\theta, \overline{5}, P_{2}}=\operatorname{diag}\left(e^{2 \pi i \frac{1}{4}} \mathbf{1}_{8}, e^{2 \pi i \frac{3}{4}} \mathbf{1}_{8}\right)
$$

Also, we achieve cancellation of twisted tadpoles at the two $\mathbf{C}^{2} / \mathbf{Z}_{\mathbf{2}}$ points fixed under $\Omega \Pi$, by locating eight D5-branes at one of them, and eight $\overline{\mathrm{D} 5}$-branes at the other, with $\gamma_{\theta^{2}, 5, Q_{1}}=\mathbf{1}_{8}, \gamma_{\theta^{2}, \overline{5}, Q_{2}}=-\mathbf{1}_{8}$. The complete spectrum for this configuration, shown in Table 9, is free of gauge and gravitational anomalies.

\footnotetext{
${ }^{6}$ Interestingly, in the non-compact contex the $\Omega \alpha$ and $\Omega \Pi$ orientifold of $\mathbf{C}^{\mathbf{2}} / \mathbf{Z}_{\mathbf{2}}$ are T-dual to two different configurations (in the spirit of [37) of type IIA NS-fivebranes, D-branes and orientifold planes, studied in [38] and [39], which nevertheless give the same massless spectrum.
} 


\begin{tabular}{|c|c|c|}
\hline Closed & Untwisted & $\mathcal{N}=1$ SUGRA $+3 \mathbf{T}+2 \mathbf{H}$ \\
\hline & Twisted & $12 \mathbf{T}+4 \mathbf{H}$ \\
\hline \multirow[t]{3}{*}{ Open } & 55 & $\mathcal{N}=1$ Vectors of $\left[S O(8)^{2}\right] \mathbf{z}_{\mathbf{4}} \times S O(8) \mathbf{z}_{\mathbf{2}}$ \\
\hline & $\overline{5} \overline{5}$ & Gauge bosons of $\left[U S p(8)^{2}\right] \mathbf{z}_{\mathbf{4}} \times U S p(8) \mathbf{z}_{\mathbf{2}}$ \\
\hline & & Fermion $_{+}(\mathbf{2 7}+\mathbf{1}, \mathbf{1} ; \mathbf{1})+(\mathbf{1}, \mathbf{2 7}+\mathbf{1} ; \mathbf{1})+(\mathbf{1}, \mathbf{1} ; \mathbf{2 7}+\mathbf{1})$ \\
\hline
\end{tabular}

Table 9: Spectrum of the $\Omega \Pi$ orientifold of $\mathbf{T}^{\mathbf{4}} / \mathbf{Z}_{\mathbf{4}}$.

\section{Tadpole cancellation in the $\mathrm{T}^{6} / \mathrm{Z}_{4}$ orientifold}

In this section we use the philosophy developed in our understanding of twisted tadpoles to solve and old puzzle in the construction and tadpole cancellation in the $\Omega$ orientifold of $\mathbf{T}^{\mathbf{6}} / \mathbf{Z}_{\mathbf{4}}$, with the A-type projection (usually called without vector structure) [9]. Here the $\mathbf{Z}_{\mathbf{4}}$ is generated by an action $\theta$ with twist $v=\frac{1}{4}(1,1,-2)$. Even though this model, which contains untwisted tadpoles, appears unrelated to our main interest in the present paper, there is an interesting relation, based on the structure of twisted tadpoles in the $\mathbf{Z}_{\mathbf{4}}$ orientifold. Specifically, it contains a twisted tadpole whose cancellation is possible only by a set of D-branes (and antibranes) completely unrelated to the untwisted tadpoles of the model. The fact that the twisted tadpole is cancelable in this manner had been overlooked (mainly due to the fact that the introduction of antibranes in orientifold constructions is relatively recent), leading to the (incorrect) claim that the $\mathbf{Z}_{\mathbf{4}}$ orientifold, with the $\mathbf{A}$-type projection is inconsistent.

Let us start reviewing the tadpoles generated by the closed string sector. and their volume dependence [9]. For convenience, we prefer to discuss the model in a T-dual version where the orientifold action is $\Omega_{3}=\Omega R_{3}(-)^{F_{L}}$. There are tadpoles for untwisted fields, generated by $\Omega_{3}$ and by $\Omega_{3} \theta^{2}$, which are proportional to the volumes $\left(V_{4} V_{1} V_{2} / V_{3}\right)^{1 / 2}$ and $\left[V_{4} /\left(V_{1} V_{2} V_{3}\right)\right]^{1 / 2}$, respectively. They are cancelled by the introduction $32 \mathrm{D}_{3^{-}}$and 32 D3-branes, whose untwisted disk diagrams lead to charges with the required volume dependence and coefficient.

The $\Omega_{3} \theta$ twisted crosscap leads to a tadpole proportional to $\sqrt{V_{4} V_{3}}$. As pointed out in [9, 10], this twisted tadpole cannot cancelled by any disk associated to the $\mathrm{D} 7_{3^{-}}$or the D3-branes in the model. However, by now we are familiar with the fact that some orientifolds require specific sets of branes, designed to cancel twisted tadpoles, even if untwisted tadpoles do not require them. With this philosophy in mind, we introduce a set of $\mathrm{D} 7_{1}-\overline{\mathrm{D}}_{1}$ pairs (one may introduce $\mathrm{D} 7_{2}-\overline{\mathrm{D}}_{2}$ pairs if desired), at the points $P$ fixed 
under $\theta$, which have coordinates $\left(z_{1}, z_{2}, z_{3}\right)$, with $z_{1}=0,(1+i) / 2, z_{2}=0,(1+i) / 2$, $z_{3}=0,1 / 2, i / 2,(1+i) / 2$. The $\theta^{2}$-twisted tadpole condition reads

$$
\operatorname{Tr} \gamma_{\theta^{2}, 7_{1}, P}-\operatorname{Tr} \gamma_{\theta^{2}, \overline{7}_{1}, P}=-16
$$

At points $Q$ fixed under $\theta^{2}$ but not under $\theta$, the condition must be

$$
\operatorname{Tr} \gamma_{\theta^{2}, 7_{1}, Q}-\operatorname{Tr} \gamma_{\theta^{2}, \overline{7}_{1}, Q}=0
$$

The closed string sector does not generated tadpoles for $\theta$-twisted fields. The cancellation of twisted disk contributions requires

$$
\sqrt{2}\left(\operatorname{Tr} \gamma_{\theta, 7_{1}, P}-\operatorname{Tr} \gamma_{\theta, \overline{7}_{1}, P}\right)-2 \operatorname{Tr} \gamma_{\theta, 7_{3}, P}-4 \operatorname{Tr} \gamma_{\theta, 3}=0
$$

At this point, we would like to recall that for the A-type projection, D7 $3^{-}$and D3branes have Chan-Paton matrices with vector structure (roughly speaking $\gamma_{\theta}^{N}=\mathbf{- 1}$ ) and $\mathrm{D}_{1^{-}}, \overline{\mathrm{D}}_{1^{-}}$-branes (and also $\mathrm{D} 7_{2^{-}}, \overline{\mathrm{D}}_{2^{-}}$-branes if introduced) have matrices with vector structure $\left(\gamma_{\theta}^{N}=+\mathbf{1}\right)$.

It is relatively easy to build models satisfying all tadpole conditions. To give an example, we proceed as follows. In order to cancel the problematic $\theta^{2}$-twisted tadpoles, we introduce $16 \mathrm{D} 7_{1}$ branes at $z_{1}=0$, and $16 \overline{\mathrm{D}}_{1}$-branes at $z_{1}=(1+i) 2$, with

$$
\gamma_{\theta, 7_{1}, 0}=\operatorname{diag}\left(e^{2 \pi i \frac{1}{4}} \mathbf{1}_{8}, e^{2 \pi i \frac{3}{4}} \mathbf{1}_{8}\right) \quad, \quad \gamma_{\theta, \overline{7}_{1},(1+i) / 2}=\operatorname{diag}\left(\mathbf{1}_{8},-\mathbf{1}_{8}\right)
$$

In order to avoid generating $\theta^{2}$-twisted tadpoles at points $Q$, not fixed under $\theta$, we introduce Wilson lines along the second complex plane, acting on the $\mathrm{D} 7_{1^{-}}, \overline{\mathrm{D}}_{1}$-branes as

$$
\gamma_{W_{2}, 7_{1}, 0}=\operatorname{diag}\left(\mathbf{1}_{4},-\mathbf{1}_{4}, \mathbf{1}_{4},-\mathbf{1}_{4}\right) \quad, \quad \gamma_{W_{2}, \overline{7}_{1},(1+i) / 2}=\operatorname{diag}\left(\mathbf{1}_{4},-\mathbf{1}_{4}, \mathbf{1}_{4},-\mathbf{1}_{4}\right)
$$

Finally, we need to introduce the set of $32 \mathrm{D}_{3^{-}}$and $32 \mathrm{D} 3$-branes to cancel the untwisted tadpoles, and constrained by (5.3). A simple choice is just

$$
\gamma_{\theta, 7_{3}}=\gamma_{\theta, 3}=\left(e^{\pi i \frac{1}{4}} \mathbf{1}_{8}, e^{\pi i \frac{3}{4}} \mathbf{1}_{8}, e^{\pi i \frac{5}{4}} \mathbf{1}_{8}, e^{\pi i \frac{7}{8}} \mathbf{1}_{8}\right)
$$

with all $\mathrm{D}_{3}$-branes sitting for instance at $z_{3}=0$, and all D3-branes sitting at $(0,0,0)$. For completeness we also provide the Chan-Paton matrices for $\Omega_{3}$

$$
\begin{aligned}
& \gamma_{\Omega_{3}, 7_{3}}=\left(\begin{array}{cccc} 
& & & \mathbf{1}_{8} \\
& & \mathbf{1}_{8} & \\
& \mathbf{1}_{8} & & \\
\mathbf{1}_{8} & & &
\end{array}\right) \quad ; \quad \gamma_{\Omega_{3}, 3}=\left(\begin{array}{lll} 
& & \\
& \mathbf{1}_{8} & \\
-\mathbf{1}_{8} & & \\
-\mathbf{1}_{8} & &
\end{array}\right) \\
& \gamma_{\Omega_{3}, 7_{1}}=\left(\begin{array}{cc} 
& \mathbf{1}_{8} \\
-\mathbf{1}_{8} &
\end{array}\right) \quad ; \quad \gamma_{\Omega_{3}, \overline{7}_{1}}=\left(\begin{array}{cc}
\varepsilon_{8} & \\
& \varepsilon_{8}
\end{array}\right)
\end{aligned}
$$


With this information it is a simple exercise to compute the resulting spectrum, which is given in Table 10. It is interesting to point out that for instance the cancellation of non-abelian anomalies for gauge factors on the D3-branes is crucially correlated with the cancellation of the problematic twisted tadpole.

Clearly, the lesson we have learned about the consistency of the $\mathbf{T}^{\mathbf{6}} / \mathbf{Z}_{\mathbf{4}}$ orientifold extends to other cases previously considered inconsistent as well, like orientifolds (with B-type projection) of $\mathbf{T}^{\mathbf{6}} / \mathbf{Z}_{\mathbf{8}}, \mathbf{T}^{\mathbf{6}} / \mathbf{Z}_{\mathbf{8}}^{\prime}$, and $\mathbf{T}^{\mathbf{6}} / \mathbf{Z}_{12}^{\prime}$, which contain the same type of twisted tadpole [9]. Hoping that the $\mathbf{Z}_{\mathbf{4}}$ example above suffices to illustrate the idea, we leave further issues for future work.

\begin{tabular}{|c|c|c|}
\hline Closed & Untwisted & $\mathcal{N}=1$ SUGRA $+1 \mathbf{L}+6 \mathbf{C}$ \\
\hline & Twisted & $32 \mathbf{C h}$ \\
\hline \multirow[t]{15}{*}{ Open } & 33 & $\mathcal{N}=1$ Vectors of $U(8)^{2}$ \\
\hline & & $\mathcal{N}=1$ Chiral $2 \times[(\mathbf{8}, \overline{\mathbf{8}})+(\mathbf{1}, \mathbf{2 8})+(\overline{\mathbf{2 8}}, \mathbf{1})]+(\mathbf{8}, \mathbf{8})+(\overline{\mathbf{8}}, \overline{\mathbf{8}})$ \\
\hline & $7_{3} 7_{3}$ & $\mathcal{N}=1$ Vectors of $U(8)^{2}$ \\
\hline & & $\mathcal{N}=1$ Chiral $2 \times[(\mathbf{8}, \overline{\mathbf{8}})+(\mathbf{1}, \mathbf{2 8})+(\overline{\mathbf{2 8}}, \mathbf{1})]+(\mathbf{8}, \mathbf{8})+(\overline{\mathbf{8}}, \overline{\mathbf{8}})$ \\
\hline & $7_{1} 7_{1}$ & $\mathcal{N}=1$ Vectors of $U(4)^{2}$ \\
\hline & & $\mathcal{N}=1$ Chiral $2 \times[(\mathbf{6}, \mathbf{1})+(\overline{\mathbf{6}}, \mathbf{1})+(\mathbf{1}, \mathbf{6})+(\mathbf{1}, \overline{\mathbf{6}})]$ \\
\hline & $\overline{7}_{1} \overline{7}_{1}$ & Gauge Bosons of $U S p(4)^{4}$ \\
\hline & & Fermion $_{+}(\mathbf{5}+\mathbf{1}, \mathbf{1}, \mathbf{1}, \mathbf{1})+(\mathbf{1}, \mathbf{5}+\mathbf{1}, \mathbf{1}, \mathbf{1})+(\mathbf{1}, \mathbf{1}, \mathbf{5}+\mathbf{1}, \mathbf{1})+(\mathbf{1}, \mathbf{1}, \mathbf{1}, \mathbf{5}+\mathbf{1})$ \\
\hline & & Cmplx. Scalars $(\mathbf{4}, \mathbf{1}, \mathbf{4}, \mathbf{1})+(\mathbf{1}, \mathbf{4}, \mathbf{1}, \mathbf{4})$ \\
\hline & & Fermion $_{-}(\mathbf{4}, \mathbf{1}, \mathbf{4}, \mathbf{1})+(\mathbf{1}, \mathbf{4}, \mathbf{1}, \mathbf{4})$ \\
\hline & $37_{3}+7_{3} 3$ & $\mathcal{N}=1$ Chiral $(\mathbf{8}, \mathbf{1} ; \mathbf{1}, \overline{\mathbf{8}})+(\mathbf{1}, \mathbf{8} ; \mathbf{1}, \mathbf{8})+(\mathbf{1}, \overline{\mathbf{8}} ; \mathbf{8}, \mathbf{1})+(\overline{\mathbf{8}}, \mathbf{1} ; \overline{\mathbf{8}}, \mathbf{1})$ \\
\hline & $37_{1}+7_{1} 3$ & $\mathcal{N}=1$ Chiral $(\overline{\mathbf{8}}, \mathbf{1} ; \mathbf{4}, \mathbf{1})+(\overline{\mathbf{8}}, \mathbf{1} ; \mathbf{1}, \mathbf{4})+(\mathbf{1}, \mathbf{8} ; \mathbf{4}, \mathbf{1})+(\mathbf{1}, \mathbf{8}, \mathbf{1}, \mathbf{4})$ \\
\hline & $7_{3} 7_{1}+7_{1} 7_{3}$ & $\mathcal{N}=1$ Chiral $(\overline{\mathbf{8}}, \mathbf{1} ; \mathbf{4}, \mathbf{1})+(\mathbf{1}, \mathbf{8} ; \mathbf{4}, \mathbf{1})$ \\
\hline & $\mathbf{7}_{3} \overline{\mathbf{7}}_{1}+\overline{\mathbf{7}}_{\mathbf{1}} \mathbf{7}_{\mathbf{3}}$ & Fermion $_{+}(\mathbf{8}, \mathbf{1} ; \mathbf{4}, \mathbf{1}, \mathbf{1}, \mathbf{1})+(\mathbf{1}, \overline{\mathbf{8}} ; \mathbf{1}, \mathbf{1}, \mathbf{4}, \mathbf{1})$ \\
\hline & & Cmplx. Scalars $(\mathbf{8}, \mathbf{1} ; \mathbf{4}, \mathbf{1}, \mathbf{1}, \mathbf{1})+(\mathbf{1}, \overline{\mathbf{8}} ; \mathbf{1}, \mathbf{1}, \mathbf{4}, \mathbf{1})$ \\
\hline
\end{tabular}

Table 10: Spectrum of a consistent $\Omega_{3}$ orientifold of $\mathbf{T}^{\mathbf{6}} / \mathbf{Z}_{\mathbf{4}}$ with $\mathbf{A}$-type projection.

\section{Phase transitions and stability regions}

The non-supersymmetric models we have been considering are absolutely stable against decay to a supersymmetric vacuum, because there is no consistent (tadpole-free) su- 
persymmetric vacuum for these orientifold projections. However, in particular regions of parameter space the configuration of branes and antibranes may become unstable and decay to other (necessarily non-BPS) systems of branes. The analysis of such decays and the stability regimes is analogous to that performed in the study of stable non-BPS states in string theory. In this section we address this issue in the simplest model we have constructed, namely the $\Omega^{\prime} \alpha$ orientifold of $\mathbf{T}^{\mathbf{4}} / \mathbf{Z}_{\mathbf{2}}$ with $\mathbf{B}$-type projection, studied in Section 2. We will take advantage of by now standard properties of brane-antibrane systems, and non-BPS branes, in $\mathbf{Z}_{\mathbf{2}}$ orbifolds (see e.g. [24, 25, 26] and references therein).

Let us consider the $\Omega^{\prime} \alpha$ orientifold of $\mathbf{T}^{\mathbf{4}} / \mathbf{Z}_{\mathbf{2}}$. Denoting the radii of the corresponding two-tori $R_{1}, R_{2}$, there are four fixed points under the $\Omega^{\prime} \alpha$ action, given by $\left(z_{1}, z_{2}\right)$ equal to $P_{1}=(0,0), P_{2}=\left(\frac{1+i}{2} R_{1}, 0\right), P_{3}=\left(0, \frac{1+i}{2} R_{2}\right)$ and $P_{4}=\left(\frac{1+i}{2} R_{1}, \frac{1+i}{2} R_{2}\right)$. Let us consider locating 8 D5-branes at $P_{1}$ and $P_{4}$ and $8 \overline{\mathrm{D} 5}$-branes at $P_{2}$ and $P_{3}$, with $\gamma_{\theta, 5}=\mathbf{1}_{8}$ and $\gamma_{\theta, \overline{5}}=-\mathbf{1}_{8}$. Table 2 provides the spectrum for this configuration, which is depicted in Figure 1. The configurations is symmetric under the exchange of the first and second complex planes, a fact which simplifies the analysis of the model.

The configuration is stable when $R_{1}$ and $R_{2}$ are large. But it may become unstable against decay to some other non-supersymmetric configuration if these geometric data change. In particular, the mass of a string stretching between the D5-branes at $P_{1}$ and the $\overline{\mathrm{D} 5}$-branes at $P_{2}$ (or between D5-branes at $P_{4}$ and $\overline{\mathrm{D} 5}$-branes at $P_{3}$ ) is

$$
M^{2}=\frac{1}{4 \pi^{2}}\left(\pi \sqrt{2} R_{1}\right)^{2}-1 / 2
$$

in $\alpha^{\prime}=1$ units. At $R_{1}=1$ the $5 \overline{5}, \overline{5} 5$ groundstates become massless, and for $R_{1}<1$ they are tachyonic, and the configuration must decay to some other nonsupersymmetric configuration, with the same charges (i.e. same contribution to the twisted and untwisted tadpoles). Let us center on this phase transition, keeping $R_{2}$ large throughout the analysis.

In the absence of the orientifold projection, the situation is reminiscent of that studied in [24], namely a set of branes and antibranes, sitting at the two fixed points in a circle modded out by a $\mathbf{Z}_{\mathbf{2}}$ action. When the fixed points come to a critical distance of $\sqrt{2}$ the tachyonic groundstate of stretched strings triggers a decay to a non-BPS D-brane wrapped on the circle. In our context, the critical distance between the fixed points is reached for $R_{1}=1$, so we may expect a decay to a set of non-BPS D6-branes (denoted $\widehat{\mathrm{D} 6}$-branes) wrapped on cycles passing through $z_{1}=0$ and $z_{1}=(1+i) / 2$.

In fact, since the branes and antibranes actually sit in a two-torus rather than in a circle, there are more tachyons than those in [24]. We obtain tachyonic modes from 

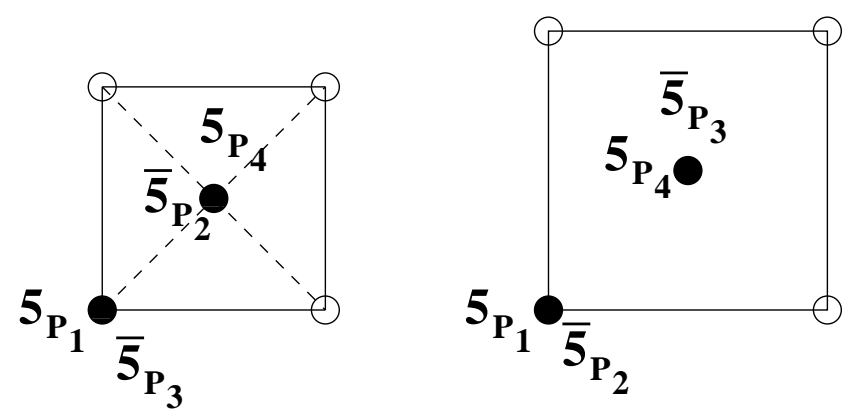

Figure 1: Configuration of D5- and $\overline{\mathrm{D} 5}$-branes in the $\Omega^{\prime} \alpha$ orientifold of $\mathbf{T}^{\mathbf{4}} / \mathbf{Z}_{\mathbf{2}}$, for $R_{1}=1$, $R_{2}>1$. Discontinuous lines denote strings stretched between D5-branes at $P_{1}$ and $\overline{\mathrm{D} 5}$-branes at $P_{2}$, which lead to tachyonic modes for $R_{1}<1$.
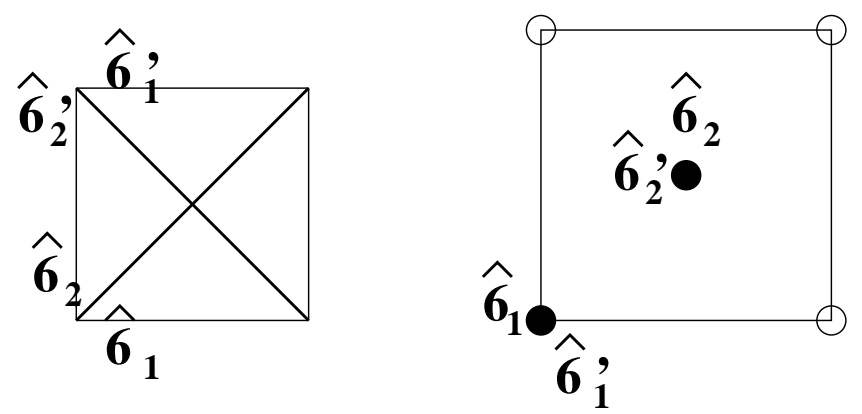

Figure 2: Configuration of $\widehat{\mathrm{D} 6}$-branes in the $\Omega^{\prime} \alpha$ orientifold of $\mathbf{T}^{\mathbf{4}} / \mathbf{Z}_{\mathbf{2}}$, for $R_{1}=1, R_{2}>1$.

strings stretching between branes and antibranes (and viceversa) in the two different directions depicted in Figure 11. Therefore we can expect the decay to a set of $\widehat{\mathrm{D} 6}$ branes will not be enough to stabilize the configuration for $R_{1}<1$. In this respect, it is useful to describe the $\widehat{\mathrm{D} 6}$-brane configuration quite explicitly, and discuss its set of light fields. We will find it indeed contains tachyonic modes for $R_{1}<1$.

Hence we consider a set of $\widehat{\mathrm{D} 6}$-branes, denoted $\widehat{\mathrm{D}}_{2}$-branes, sitting at $z_{2}=0$, and partially wrapped on the first plane, so that they pass through the points $(0,0)$, $\left(\frac{1+i}{2} R_{1}, 0\right)$, and another set of $\widehat{\mathrm{D} 6}$-branes, denoted $\widehat{\mathrm{D}}_{2}$-branes, sitting at $z_{2}=\frac{1+i}{2} R_{2}$, and partially wrapped on the first plane so that they pass through $\left(0, \frac{1+i}{2} R_{2}\right),\left(\frac{1+i}{2} R_{1}, \frac{1+i}{2} R_{2}\right)$. We also need to introduce their $\Omega^{\prime} \alpha$ images, denoted with a prime. The complete configuration is depicted in Figure 2 .

In order for this configuration to have the same twisted charges as the previous one, we must take $\gamma_{\hat{6}_{1}}=\mathbf{1}_{4}, \gamma_{\hat{6}_{2}}=\mathbf{1}_{4}$. The contribution of $\widehat{\mathrm{D} 6}$-branes and $\widehat{\mathrm{D} 6}{ }^{\prime}$-branes to the twisted tadpoles at each fixed point add up and cancel the contribution from the 
closed string sector.

Notice that the relation between tensions of the fractional D5-, $\overline{\mathrm{D} 5}$-branes, and the truncated $\widehat{\mathrm{D} 6}$-branes is 26, 24] $T_{\hat{6}}=\frac{\sqrt{2}}{2 \pi} T_{5}$. The total tension of the 8 six-branes after compactification on the $\mathbf{T}^{4}$ is $8 \times 2 \pi \times \sqrt{2} R_{1} \times T_{\hat{6}}=16 R_{1} T_{5}$, and for $R_{1}=1$ agrees with the tension of the 8 D5- plus $8 \overline{\mathrm{D} 5}$-branes in the initial configuration (Fig. 囵), making the transition energetically possible.

However, the configuration of $\widehat{\mathrm{D} 6}$-branes is actually unstable for $R_{1}<1$ due to the tachyonic groundstate of e.g. a $\hat{6}_{1} \hat{6}_{1}$ string stretching between the points $\left(\frac{1+i}{2} R_{1}, 0\right)$ and $\left(R_{1}, 0\right)$. Also, it is unstable for $R_{1}>1$ due to a tachyonic momentum mode e.g. along the $\widehat{\mathrm{D}}_{1}$-brane. In any event, it is an interesting exercise to obtain the chiral spectrum on this set of $\widehat{\mathrm{D} 6}$-branes, which is marginally tachyon-free for $R_{1}=1$, and verify the cancellation of anomalies in the effective six-dimensional field theory.

The spectrum in the $\hat{6}_{1} \hat{6}_{1}$ sector can be extracted from the computations in [24]. Notice that $\Omega^{\prime} \alpha$ maps this sector to the $\hat{6}_{1}^{\prime} \hat{6}_{1}^{\prime}$, so we may keep just the former and not impose the orientifold projection. We obtain gauge bosons of $U(4)$, and two righthanded fermions in the adjoint representation. We also need to compute the $\hat{6}_{1} \hat{6}_{1}^{\prime}$ sector, which is mapped to itself under $\Omega^{\prime} \alpha$, and so suffers the orientifold projection. An important observation is that, since these branes intersect twice, the spectrum appears in two copies. In the NS sector, each intersection would give rise to tachyonic scalars, but they are projected out by the $\mathbf{Z}_{\mathbf{2}}$ orbifold projection ๆ. In the R sector, the orbifold projection leaves one right-handed fermion (per intersection) transforming in the adjoint of $U(4)$, and the orientifold projection reduces it to the two-index antisymmetric representation $\mathbf{6}$. The $\hat{6}_{1}^{\prime} \hat{6}_{1}$ sector also gives two right-handed fermions in the 6. Finally, $\widehat{\mathrm{D}}_{2^{-}}$and $\widehat{\mathrm{D}}_{2}^{\prime}$ branes lead to the analogous spectrum. The complete open string spectrum is provided in Table 11. The model is free of gauge and gravitational anomalies. Recall that, as mentioned above, stretched strings and momentum modes lead to an additional set of marginally massless scalars, not shown in Table 11, which become tachyonic/massive at $R_{1} \neq 1$.

The tachyonic momentum modes present at $R_{1}>1$ clearly signal an instability against decay to the original configuration of D5- $\overline{\mathrm{D} 5}$ pairs. Let us instead consider the regime $R_{1} \leq 1$, and look for a non-BPS configuration stable in that situation. Before the orientifold projection, the initial configuration consists of a set of branes and antibranes at fixed points in a square $\mathbf{T}^{\mathbf{2}} / \mathbf{Z}_{\mathbf{2}}$. The phase transition when the

\footnotetext{
${ }^{7}$ These tachyons would parametrize the possibility of recombining the two intersecting $\widehat{\mathrm{D} 6}$-branes into smooth ones, which would no longer pass through the fixed point. This is clearly forbidden by conservation of $\mathbf{Z}_{\mathbf{2}}$ twisted charges, so the $\mathbf{Z}_{\mathbf{2}}$ projection removes the tachyons.
} 


\begin{tabular}{|c||c|}
\hline $\mathbf{6 6}, \mathbf{6}^{\prime} \mathbf{6}^{\prime}$ & Gauge bosons of $U(4)^{2}$ \\
\hline & Fermion $_{+} 2 \times[(\mathrm{Adj}, 1)+(1, \mathrm{Adj})]$ \\
\hline $\mathbf{6 6}^{\prime}$ & Fermion $_{+} 2 \times[(6,1)+(1,6)]$ \\
\hline $\mathbf{6}^{\prime} \mathbf{6}$ & Fermion $_{+} 2 \times[(6,1)+(1,6)]$ \\
\hline
\end{tabular}

Table 11: Open string spectrum for the configuration of $\widehat{\mathrm{D} 6}$-branes in Figure 2 .
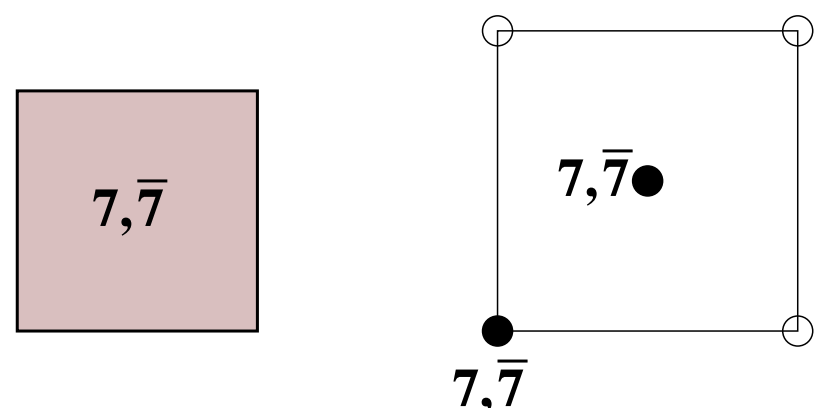

Figure 3: Configuration of D7-D7pairs in the $\Omega^{\prime} \alpha$ orientifold of $\mathbf{T}^{\mathbf{4}} / \mathbf{Z}_{\mathbf{2}}$, for $R_{1}=1$. The branes wrap the first (shaded) complex plane, and sit at the dot locations in the second.

size of the two-torus shrinks, is analogous to that studied in [25]. In our context, we would obtain a set of D7- $\overline{\mathrm{D} 7}$ pairs wrapped on the first plane, and with suitable order two Wilson lines turned on in order to reproduce the correct charges at the fixed points. At this point a question arises, since the orientifold projection $\Omega^{\prime} \alpha$ projects out the 8-form under which D7-branes are charged, so it seems that these should not be allowed in the model. The resolution is simply that the orientifold projection actually maps D7-branes to $\overline{\mathrm{D} 7}$-branes and viceversa, so that the object formed by a pair of D7- $\overline{\mathrm{D}} 7$ branes exchanged by the orientifold projection, is indeed an allowed state in the orientifolded theory. Namely this state is neutral under the 8-form, so its existence is consistent with the latter being projected out. This non-BPS $\widehat{\mathrm{D} 7}$-brane is analogous to the non-BPS D7-brane of type I string theory [40] (but not suffering its stability problems [41], due to the absence of background branes in the model).

Hence the initial configuration of D5- $\overline{\mathrm{D} 5}$ branes decays, for $R_{1}<1$, to two sets of D7- $\overline{\mathrm{D} 7}$ pairs (or $\widehat{\mathrm{D} 7}$-branes), wrapped on the first plane, and sitting at the locations $z_{2}=0$ and $z_{2}=\frac{(1+i)}{2} R_{2}$ in the second. The configuration is schematically shown in figure 3 .

In order to reproduce the correct twisted charges at the $\alpha$-fixed points, we need to 
take

$$
\gamma_{\theta, 7_{1}}=e^{\pi i \frac{1}{2}} \mathbf{1}_{8} \quad ; \quad \gamma_{\theta, \overline{7}_{1}}=e^{\pi i \frac{3}{2}} \mathbf{1}_{8}
$$

Notice that D7-branes have Chan-Paton matrices 'without vector structure' In order to have zero contribution to the tadpoles at $\theta$-fixed points not fixed under $\Omega^{\prime} \alpha$, we introduce the Wilson lines

$$
\gamma_{W_{1}, 7}=\operatorname{diag}\left(\mathbf{1}_{4},-\mathbf{1}_{4}\right) \quad ; \quad \gamma_{W_{1}, \overline{7}}=\operatorname{diag}\left(\mathbf{1}_{4},-\mathbf{1}_{4}\right)
$$

The tension of D7-branes is related to that of D5-branes by $T_{7}=\frac{1}{4 \pi^{2}} T_{5}$, 42 , so the total tension of the sevenbranes, after compactification on the torus is $16 \times$ $\left(2 \pi R_{1}\right)^{2} T_{7}=16 \times R_{1}^{2} T_{5}$, and for $R_{1}=1$ equals the tension of the 8 D5- $\overline{\mathrm{D} 5}$ pairs in the initial model.

Let us describe the resulting spectrum, centering on the set of branes at $z_{2}=0$. The 77 sector is mapped to the $\overline{7} \overline{7}$ sector by the orientifold action, so we keep the former and perform no projection. We obtain $\mathcal{N}=1$ vector multiplets of $U(4)^{2}$, i.e. gauge bosons and right handed fermions. The $7 \overline{7}$ sector is mapped to itself by the orbifold and orientifold projections. The tachyonic NS groundstate survives the (opposite) GSO projection in this sector, but the $\mathbf{Z}_{2}$ twist projects it out. In the $\mathrm{R}$ sector, we obtain right-handed fermions which, after the orientifold projection, transform in the antisymmetric representations, $(\mathbf{6}, \mathbf{1})+(\mathbf{1}, \mathbf{6})$. The $\overline{7} 7$ spectrum is obtained analogously. Finally, the set of branes at $z_{2}=(1+i) / 2$ give another copy of the fields above. The

\begin{tabular}{|c|c|}
\hline $77, \overline{7} \overline{7}$ & Gauge bosons of $U(4)^{4}$ \\
\hline & Fermion $_{+}(\operatorname{Adj}, \mathbf{1}, \mathbf{1}, \mathbf{1})+(\mathbf{1}, \operatorname{Adj}, \mathbf{1}, \mathbf{1})+(\mathbf{1}, \mathbf{1}, \operatorname{Adj}, \mathbf{1})+(\mathbf{1}, \mathbf{1}, \mathbf{1}, \operatorname{Adj})$ \\
\hline $7 \overline{7}$ & Fermion $_{+}(\mathbf{6}, \mathbf{1}, \mathbf{1}, \mathbf{1})+(\mathbf{1}, \mathbf{6}, \mathbf{1}, \mathbf{1})+(\mathbf{1}, \mathbf{1}, \mathbf{6}, \mathbf{1})+(\mathbf{1}, \mathbf{1}, \mathbf{1}, \mathbf{6})$ \\
\hline$\overline{7} 7$ & Fermion $_{+}(\mathbf{6}, \mathbf{1}, \mathbf{1}, \mathbf{1})+(\mathbf{1}, \mathbf{6}, \mathbf{1}, \mathbf{1})+(\mathbf{1}, \mathbf{1}, \mathbf{6}, \mathbf{1})+(\mathbf{1}, \mathbf{1}, \mathbf{1}, \mathbf{6})$ \\
\hline
\end{tabular}
complete open string spectrum is shown in Table 12

Table 12: Open string spectrum for the configuration of D7- $\overline{\mathrm{D} 7}$ branes in Figure 3 .

Obviously, due to the symmetry between the two complex planes, a similar set of transitions is obtained by varying $R_{2}$, while keeping $R_{1}$ large, so we need not repeat

\footnotetext{
${ }^{8}$ This can be derived by analyzing the $\mathbf{Z}_{2}$ projection in 57 sectors, which exist at $R_{1}=1$ if for instance only part of the D5- $\overline{\mathrm{D} 5}$ branes are transformed into $\widehat{\mathrm{D} 7}$-branes. The result then follows for $R_{1}<1$.
} 
the analysis. However, there are new transitions if we make both $R_{1}$ and $R_{2}$ small, which we study in what follows. Let us keep $R_{1} \leq 1$, so that the initial configuration is provided by the set of $\widehat{\mathrm{D} 7}$-branes we have just studied, and make $R_{2}$ small. At $R_{2}=1$ the groundstate of a string stretching between e.g. a D7-brane at $z_{2}=0$ and a $\overline{\mathrm{D} 7}$-brane at $z_{2}=(1+i) / 2$ becomes massless, and leads to a tachyon for $R_{2}<1$.

The transition is very similar to those considered previously, so our discussion is more sketchy. We may consider a configuration of 8 non-BPS $\widehat{\mathrm{D} 8}$-branes completely wrapped on the first plane and partially wrapped on the second, and their $8 \Omega^{\prime} \alpha$ images, denoted $\widehat{\mathrm{D}^{\prime}}$-branes. Such configuration would be stable only at $R_{2}=1$. In order to reproduce the correct twisted charges, the orbifold and Wilson lines ChanPaton matrices must be

$$
\begin{gathered}
\gamma_{\theta, \hat{8}}=\mathbf{1}_{8} \quad ; \quad \gamma_{W_{1}, \hat{8}}=\operatorname{diag}\left(\mathbf{1}_{4},-\mathbf{1}_{4}\right) \\
\gamma_{\theta, \hat{8}^{\prime}}=\mathbf{1}_{8} \quad ; \quad \gamma_{W_{1}, \hat{8}^{\prime}}=\operatorname{diag}\left(\mathbf{1}_{4},-\mathbf{1}_{4}\right)
\end{gathered}
$$

This configuration is actually T-dual to a configuration of $\widehat{\mathrm{D} 6}$-branes identical to that considered previously (up to a relabeling of the first and second complex planes). The open string spectrum is therefore identical to that given in Table 11, replacing 6 and $6^{\prime}$ by 8 and $8^{\prime}$.

The stable configuration for $R_{1}<1, R_{2}<1$ is provided by a set of 16 D9- and $16 \overline{\mathrm{D} 9}$ branes. It is easy to see that the total tension of these objects agrees, for $R_{1}=R_{2}=1$, with the total tension of the 8 D5- and $8 \overline{\mathrm{D} 5}$-branes of the initial configuration, or, for $R_{2}=1$ and arbitrary $R_{1}<1$, with the configuration of 8 D7-D7pairs, so the transition is energetically allowed precisely when the tachyon develops.

In order to obtain the correct twisted charges, the choice of twist and Wilson line Chan-Paton matrices is

$$
\begin{array}{ccc}
\gamma_{\theta, 9}=\mathbf{1}_{16} & \gamma_{W_{1}, 9}=\mathbf{1}_{16} & \gamma_{W_{2}, 9}=\operatorname{diag}\left(\mathbf{1}_{8},-\mathbf{1}_{8}\right) \\
\gamma_{\theta, \overline{9}}=-\mathbf{1}_{16} & \gamma_{W_{1}, \overline{9}}=-\mathbf{1}_{16} & \gamma_{W_{2}, \overline{9}}=\operatorname{diag}\left(\mathbf{1}_{8},-\mathbf{1}_{8}\right)
\end{array}
$$

This configuration is easily seen to be T-dual to a configuration with eight D5- $\overline{\mathrm{D} 5}$ pairs distributed exactly as in the configuration we started with. Hence, the computation of the spectrum leads to the states in Table 2, with fivebranes replaced by ninebranes.

In Figure 1 we combine the results of this section in a phase diagram of this model, for the two-dimensional slice of parameter space we have studied. 


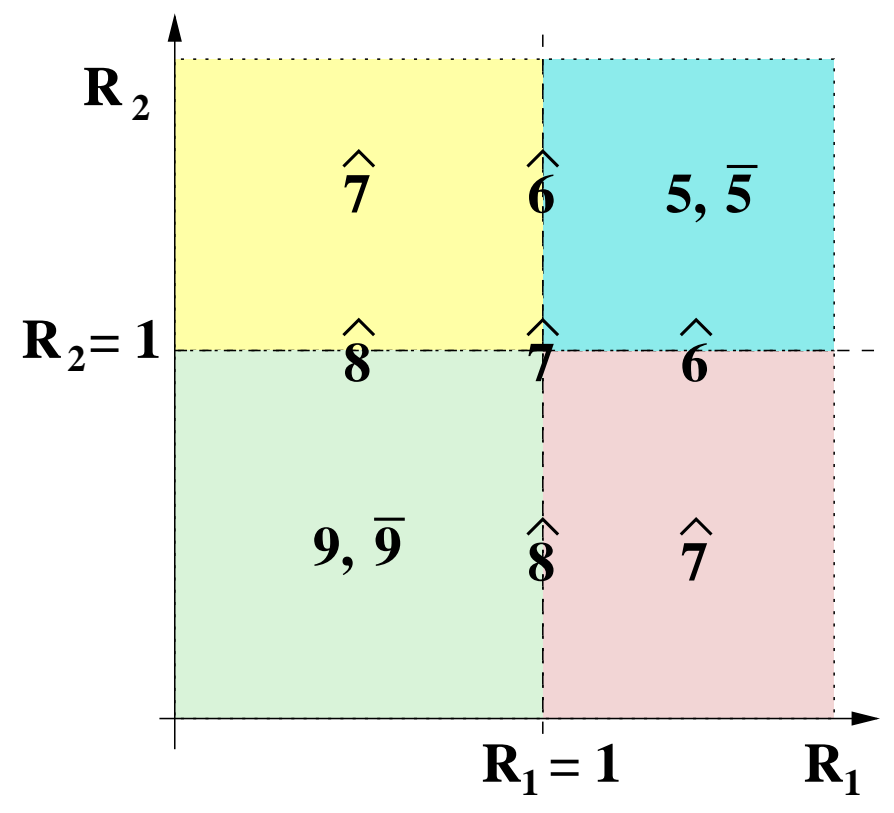

Figure 4: Phase diagram for the $\Omega^{\prime} \alpha$ orientifold of $\mathbf{T}^{\mathbf{4}} / \mathbf{Z}_{\mathbf{2}}$. For large $R_{1}, R_{2}$ the stable configuration corresponds to a set of D5- and $\overline{\mathrm{D} 5}$-branes at orbifold fixed points. When one of the radii decreases below the critical value $R=1$, brane-antibrane pairs decay into non-BPS $\widehat{\mathrm{D} 7}$-branes, wrapped in the small two-torus and transverse to the large one. For $R_{1}, R_{2}<1$, the stable configuration contains a set of D9- and $\overline{\mathrm{D} 9}$-branes. When one or two of the radii are exactly equal to the critical value, there exist marginally tachyon-free configurations. For instance, configurations of non-BPS $\widehat{D 6}$-branes partially wrapping a 1cycle in the first plane, and transverse to the second, for $R_{1}=1, R_{2}>1$; configurations of non-BPS $\widehat{\mathrm{D}} 8$-branes partially wrapping a 1-cycle in the first plane, and completely wrapped on the second, for $R_{1}=1, R_{2}<1$; and a configuration of non-BPS $\widehat{\mathrm{D} 7}$-branes wrapping one 1-cycle on each plane for $R_{1}=1, R_{2}=1$. 


\section{Conclusions}

In this paper we have studied families of type IIB orientifolds whose closed string sector does not generate untwisted tadpoles, but generates non-zero twisted tadpoles. In order to render the models consistent, the required open string sector must correspond to nonsupersymmetric configurations of branes. We have discussed how to use pairs of branes and antibranes, and non-BPS D-branes to saturate such tadpoles. The models cannot decay to any supersymmetric vacuum, and have a rich structure of phase transitions as the geometry of internal space is varied, which we have explored in detail in a particular example (leaving the dynamics of geometric moduli aside). We hope this type of analysis is useful in understanding the dynamics of brane-antibrane systems in more interesting compactifications, like the semirealistic models in [17, 20].

Our analysis is related to a more general question in the orientifold construction: Given a consistent orientifold action on the closed string sector of a type IIB compactification, is it always possible to find a suitable open string sectors cancelling the closed string tadpoles? The answer is clearly 'no' if one insists on introducing open string sectors preserving the supersymmetries which are unbroken in the closed sector. Relaxing this condition, by introducing antibranes or non-BPS branes in the construction, leads however to cancellation of many problematic tadpoles. For instance, untwisted tadpoles requiring a net negative D-brane charge [15, 16, 18, 10, 11, or, as explored in 19] and in the present paper, twisted tadpoles cancelable only by D-branes for which no untwisted tadpole exists. In this respect, we have discussed how the $\mathbf{Z}_{\mathbf{4}}$ orientifold without vector structure in [9] (and presumably other related examples), previously thought inconsistent, can be consistently completed by introducing brane-antibrane pairs specifically designed to cancel the problematic twisted tadpoles. We find these results are encouraging and illustrate the power of brane-antibrane configurations (and in general, non-BPS brane configurations) in cancelling closed string tadpoles.

On the other hand, there is no general theorem ensuring this must always be the case. Thinking in terms of the space of $R R$ charges in a given model, there is no guarantee that the closed string sector will define a vector of orientifold charges for which a suitable state (brane configuration) in string theory exists. In fact, we have found one example for which the kind of non-BPS configurations considered in the present paper is not enough to achieve tadpole cancellation. The orientifold charges lie on an empty site in the charge lattice, at least as far as the brane configurations in the present paper are concerned. Whether more general configurations provide states with the correct charges or not, remains an interesting open question deserving further 
study.

\section{Acknowledgements}

It is a pleasure to thank G. Aldazabal, E. Eyras, J. Gomis, L. E. Ibáñez, and F. Quevedo for useful conversations. R. R. thanks the Theory Division at CERN, and A. U. thanks G. Aldazabal and the Centro Atómico Bariloche (Argentina), for hospitality during the completion of this work. A. U. is grateful to M. González for encouragement and support.

\section{A Miscelanea}

\section{A.1 Chan-Paton matrices}

In $\Omega^{\prime} \alpha$ orientifolds, the general form of the Chan-Paton matrices satisfying the algebraic consistency conditions (group law), and defining a gauge bundle without vector structure (roughly speaking verifying $\gamma_{\theta}^{N}=\mathbf{- 1}$ ) are of the form

$\gamma_{\theta}=\operatorname{diag}\left(e^{\frac{i \pi}{N}} 1_{n_{1}}, e^{\frac{i \pi 3}{N}} 1_{n_{2}}, \ldots, e^{\frac{i \pi(2 P-1)}{N}} 1_{n_{P}}, e^{\frac{i \pi(2 P+1)}{N}} 1_{n_{P+1}}, \ldots, e^{\frac{i \pi(4 P-3)}{N}} 1_{n_{N-1}}, e^{\frac{i \pi(4 P-1)}{N}} 1_{n_{N}}\right)$

The orientifold projection $\Omega^{\prime} \alpha$ implies $n_{i}=n_{N+1-i}$. The structure of the orientifold actions on Chan-Paton indices is analogous to that determined in [27. For D5-branes (in Section 2) and D3-branes (in Section 3) the Chan-Paton orientifold action is

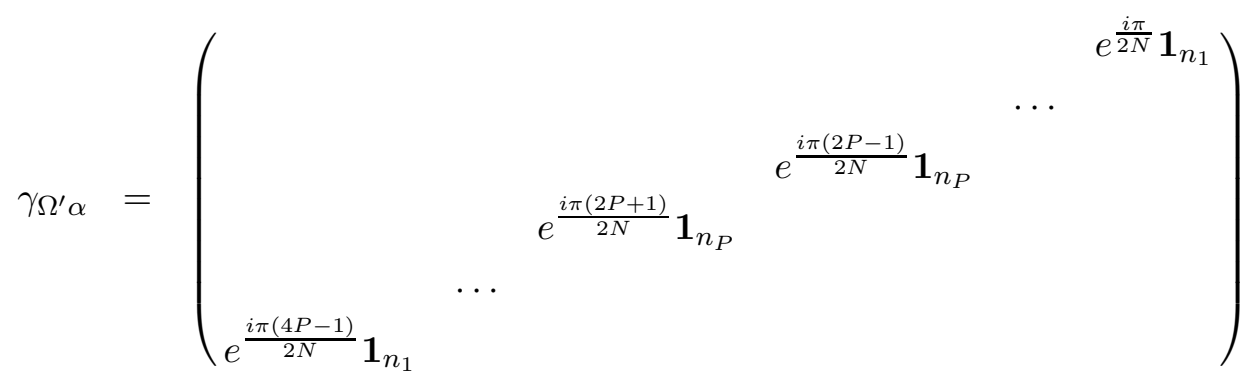

Since $\Omega^{2}=-1$ in 95 and $37_{i}$ sectors [5], the orientifold Chan-Paton action on D9- (in Section 2) and $\mathrm{D} 7_{i}$-branes (in Section 3 ) is given by an 'antisymmetrized' version of the above matrix, namely

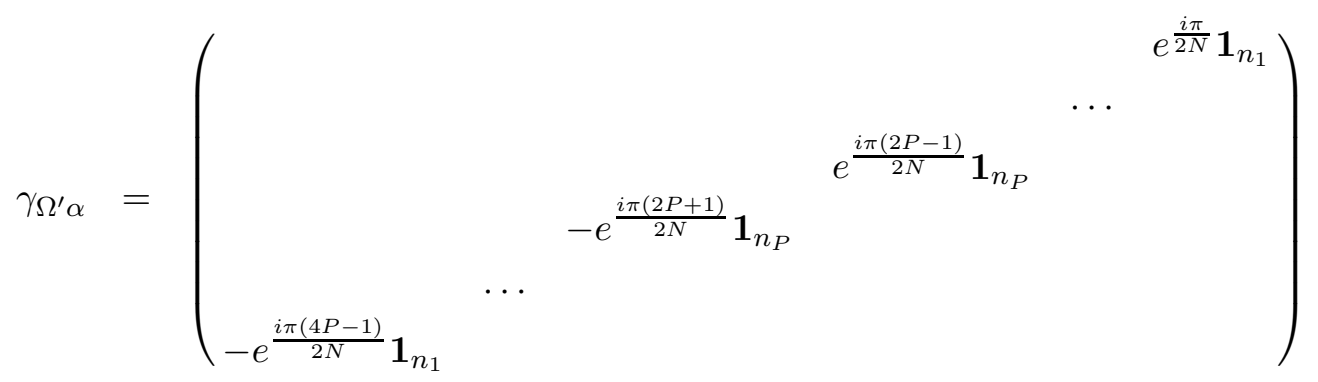

These matrices satisfy

$$
\operatorname{Tr}\left(\gamma_{\Omega^{\prime} \alpha^{2 k+1}}^{T} \gamma_{\Omega^{\prime} \alpha}^{-1}\right)= \pm \operatorname{Tr} \gamma_{\theta^{2 k+1}}
$$


with the positive and negative signs for (A.2) and (A.3), respectively.

Matrices satisfying the algebraic constraints, and defining a Chan-Paton bundle with vector structure, are of the form

$$
\gamma_{\theta}=\operatorname{diag}\left(\mathbf{1}_{n_{0}}, e^{2 \pi i \frac{1}{N}} \mathbf{1}_{n_{1}}, \ldots, e^{2 \pi i \frac{N-1}{N}} \mathbf{1}_{n_{N-1}}\right)
$$

The orientifold projection imposes $n_{N-i}=n_{i}$. The $\Omega^{\prime} \alpha$ Chan-Paton action for D5branes (in section 2) and D3-branes (in Section 3) is

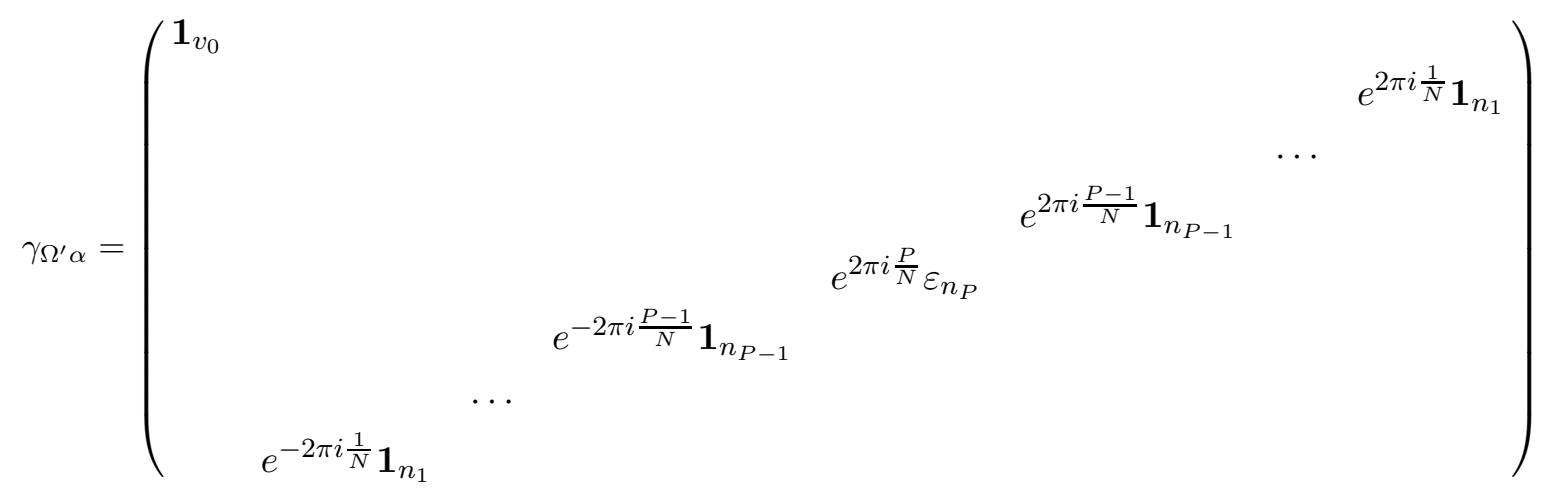

For D9-branes (Section 2) and D7 ${ }_{i}$-branes (Section 3) the action must be

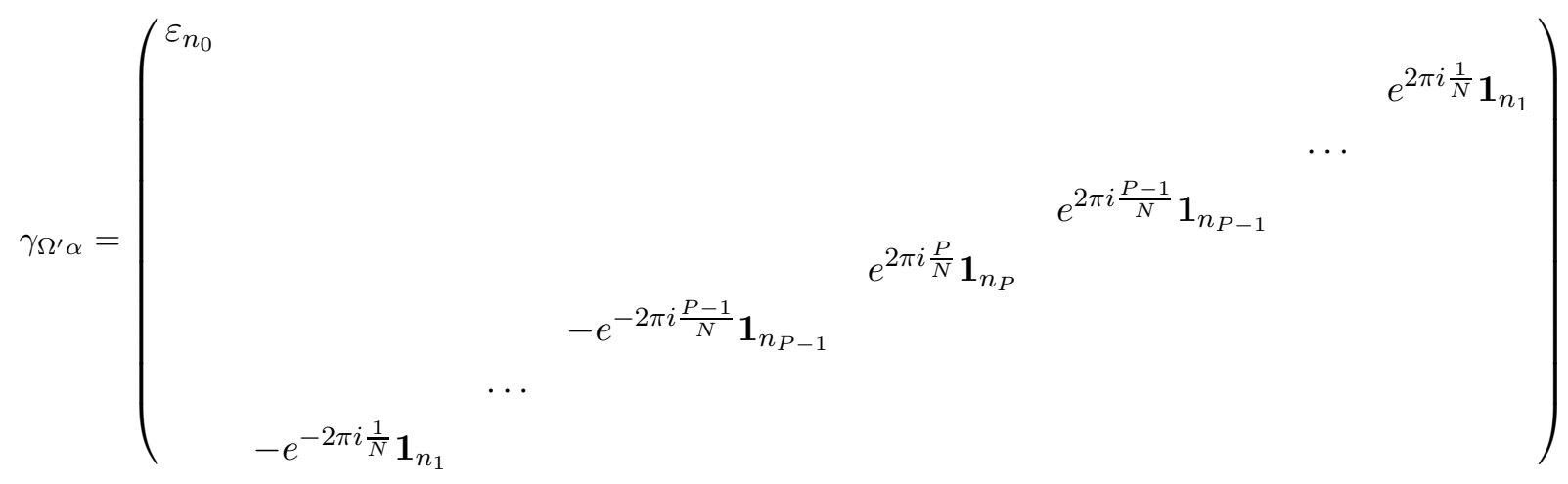

They satisfy

$$
\operatorname{Tr}\left(\gamma_{\Omega^{\prime} \alpha^{2 k+1}}^{T} \gamma_{\Omega^{\prime} \alpha}^{-1}\right)= \pm \operatorname{Tr} \gamma_{\theta^{2 k+1}}
$$

with the positive and negative signs for (A.6) and (A.7), respectively.

\section{A.2 Tadpole computations}

Tadpoles for the specific four-dimensional models studied in Section 3 have not appeared in the literature, but are easily computed following standard techniques (see e.g. the appendix in [9] for a general discussion for four-dimensional orientifolds, also [43, 8]). The only modifications are analogous to those in the six-dimensional case, which have been discussed in [27]. In order to keep track of the twisted charge normalization of crosscaps with respect to disks, we will introduce a set of D3-branes, and 
compute the one-loop amplitudes. After factorization, crosscap and disk contributions can be directly read. Disks for other types of D-branes are obtained analogously.

The twisted contributions of the cylinder, Möbius strip and Klein bottle in the factorization limit are

$$
\begin{aligned}
\mathcal{C} & =\sum_{k=1}^{N-1} \mathcal{C}\left(\theta^{k}\right)=\sum_{k=1}^{N-1} \prod_{i=1}^{3}\left(2 \sin \left(\pi k v_{i}\right)\right)\left(\operatorname{Tr} \gamma_{\theta^{k}, 3}\right)^{2} \\
\mathcal{M} & =\mathcal{M}\left(\Omega^{\prime} \alpha^{2 k+1}\right)=\sum_{k=0}^{N-1} 8 \times \prod_{i=1}^{3} 2 \sin \left(\pi(2 k+1) v_{i} / 2\right) \operatorname{Tr}\left(\gamma_{\Omega^{\prime} \alpha^{2 k+1}}^{T} \gamma_{\Omega^{\prime} \alpha^{2 k+1}}^{-1}\right) \\
\mathcal{K} & =\sum_{k=0}^{N-1}\left[\mathcal{K}\left(1, \Omega^{\prime} \alpha^{2 k+1}\right)+\mathcal{K}\left(\theta^{N / 2}, \Omega^{\prime} \alpha^{2 k+1}\right)\right]= \\
& =\sum_{k=0}^{N-1} 16\left[\prod_{i=1}^{3} \frac{2 \sin \left(\pi(2 k+1) v_{i}\right)}{4 \cos ^{2}\left(\pi(2 k+1) v_{i} / 2\right)} \pm \frac{2 \sin \left(\pi(2 k+1) v_{3}\right)}{4 \cos ^{2}\left(\pi(2 k+1) v_{3} / 2\right)}\right]
\end{aligned}
$$

with the sign in the Klein bottle is positive (negative) for the $\mathbf{A}$ - type (B-type) projection.

The cylinder can be recast as

$$
\mathcal{C}=\sum_{k=1}^{N-1} \frac{1}{\prod_{i=1}^{3} 2 \sin \left(\pi k v_{i}\right)}\left[\prod_{i=1}^{3}\left[2 \sin \left(\pi k v_{i}\right)\right] \operatorname{Tr} \gamma_{\theta^{k}, 3}\right]^{2}
$$

The matrices in section A.1 satisfy

$$
\operatorname{Tr}\left(\gamma_{\Omega^{\prime} \alpha^{2 k+1}, 3}^{T} \gamma_{\Omega^{\prime} \alpha^{2 k+1,3}}^{-1}\right)= \pm \operatorname{Tr}\left(\gamma_{\Omega^{\prime} \alpha^{2 k+1+N}, 3}^{T} \gamma_{\Omega^{\prime} \alpha^{2 k+1+N}, 3}^{-1}\right)=\operatorname{Tr} \gamma_{\theta^{2 k+1}, 3}
$$

with the upper (lower) sign for matrices with (without) vector structure, which for D3-branes is correlated with the $\mathbf{B}$-type (A-type) projection. This allows to rewrite the Moebius strip contribution as

$$
\mathcal{M}=\sum_{k=0}^{N / 2-1} \frac{1}{8 s_{1} s_{2} s_{3}} 2 \times\left[8 s_{1} s_{2} s_{3} \operatorname{Tr} \gamma_{\theta^{2 k+1}, 3}\right]\left[32\left(\tilde{s}_{1} \tilde{s}_{2} \tilde{s}_{3} \pm \tilde{c}_{1} \tilde{c}_{2} \tilde{s}_{3}\right)\right]
$$

with the upper (lower) for the A-type (B-type) projection (notice the additional sign flip in shifting $k \rightarrow k+N / 2$ in the trigonometric factors). Also we have introduced

$$
\begin{aligned}
& s_{i}=\sin \left(\pi(2 k+1) v_{i}\right), \quad \tilde{s}_{i}=\sin \left(\pi(2 k+1) v_{i} / 2\right), \\
& c_{i}=\cos \left(\pi(2 k+1) v_{i}\right), \quad \tilde{c}_{i}=\cos \left(\pi(2 k+1) v_{i} / 2\right)
\end{aligned}
$$

Finally, the Klein bottle can be rewritten as

$$
\begin{aligned}
\mathcal{K} & =\sum_{k=0}^{N / 2-1} 16 \frac{1}{8 s_{1} s_{2} s_{3}}\left[\frac{8 s_{1} s_{2} s_{3} \tilde{s}_{1} \tilde{s}_{2} \tilde{s}_{3}}{\tilde{c}_{1} \tilde{c}_{2} \tilde{c}_{3}}+\frac{8 s_{1} s_{2} s_{3} \tilde{c}_{1} \tilde{c}_{2} \tilde{s}_{3}}{\tilde{s}_{1} \tilde{s}_{2} \tilde{c}_{3}} \pm \frac{16 s_{1} s_{2} s_{3} \tilde{s}_{3}}{\tilde{c}_{3}}\right]= \\
& =\sum_{k=0}^{N / 2-1} \frac{1}{8 s_{1} s_{2} s_{3}}\left[32\left(\tilde{s}_{1} \tilde{s}_{2} \tilde{s}_{3} \pm \tilde{c}_{1} \tilde{c}_{2} \tilde{c}_{3}\right)\right]^{2}
\end{aligned}
$$


with the upper (lower) sign for the $\mathbf{A}$-type (B-type) projection.

The total contribution $\mathcal{C}+\mathcal{M}+\mathcal{K}$ factorizes as

$$
\begin{array}{cl}
\sum_{k=0}^{N-1} \frac{1}{\prod_{i=1}^{3} 2 \sin \left(\pi k v_{i}\right)}\left[\prod_{i=1}^{3} 2 \sin \left(\pi k v_{i}\right) \operatorname{Tr} \gamma_{\theta^{k}, 3}\right. & + \\
+32 \delta_{k, 1} \bmod 2 \sin \left(\pi k v_{3} / 2\right)\left(\sin \left(\pi k v_{1} / 2\right) \sin \left(\pi k v_{2} / 2\right)\right. & \left.\left. \pm \cos \left(\pi k v_{1} / 2\right) \cos \left(\pi k v_{2} / 2\right)\right)\right]^{2}
\end{array}
$$

from which, after use of trigonometric identities, we can read off the crosscap tadpoles

$$
\begin{array}{ll}
\mathcal{T}_{k}=-16 \delta_{k, 1 \bmod 2}\left(\sin \left(\pi k v_{1}\right)+\sin \left(\pi k v_{2}\right)\right) & \text { for the } \mathbf{A}-\text { type projection } \\
\mathcal{T}_{k}=+16 \delta_{k, 1 \bmod 2 \sin \left(\pi k v_{3}\right)} & \text { for the } \mathbf{B}-\text { type projection }
\end{array}
$$

in units in which the D3-brane disk tadpole is $\prod_{i} 2 \sin \left(\pi k v_{i}\right) \operatorname{Tr} \gamma_{\theta^{k}, 3}$. It is straightforward to check (for instance, from $37_{i}$ cylinders) that in these units the twisted charges of a $\mathrm{D} 7_{i}$-brane is $2 \sin \left(\pi k v_{i}\right) \operatorname{Tr} \gamma_{\theta^{k}, 7_{i}}$.

\section{References}

[1] A. Sagnotti in Cargese' 87 "Non-perturbative Quantum Field Theory", ed. G. Mack et al. (Pergamon Press 88), pag. 521; "Some properties of open string theories", hep-th/9509080.

[2] J. Dai, R. G. Leigh, J. Polchinski, "New connections between string theories", Mod. Phys. Lett. 4 (1989) 2073; R. G. Leigh, "Dirac-Born-Infeld Action From Dirichlet Sigma Model", Mod. Phys. Lett. 4 (1989) 2767.

[3] P. Horava, 'Strings on world-sheet orbifolds', Nucl. Phys. B327 (1989) 461; "Background duality of open string models", Phys. Lett. B231 (1989) 251; "Twodimensional stringy black holes with one asymptotically flat domain", Phys. Lett. B289 (1992) 293; "Equivariant topological sigma models", Nucl. Phys. B418 (1994) 571.

[4] G. Pradisi, A. Sagnotti, "Open strings orbifolds", Phys. Lett. B216 (1989) 59; M. Bianchi, A. Sagnotti, "On the systematics of open string theories", Phys. Lett. B247 (1990) 517; "Twist symmetry and open string Wilson lines", Nucl. Phys. B361 (1991) 519.

[5] E. Gimon, J. Polchinski, 'Consistency conditions for orientifolds and D manifolds', Phys. Rev. D54 (1996) 1667, hep-th/9601038. 
[6] E. G. Gimon, C. V. Johnson, 'K3 orientifolds', Nucl. Phys. B477 (1996) 715, hepth/9604129; A. Dabholkar, J. Park, 'Strings on orientifolds', Nucl. Phys. B477 (1996) 701, hep-th/9604178.

[7] C. Angelantonj, M. Bianchi, G. Pradisi, A. Sagnotti, Y. S. Stanev, 'Chiral asymmetry in four-dimensional open string vacua', Phys.Lett. B385 (1996) 96, hepth/9606169; M. Berkooz, R. G. Leigh, 'A $D=4 \mathcal{N}=1$ orbifold of type I strings', Nucl.Phys. B483 (1997) 187, hep-th/9605049; Z. Kakushadze, 'Aspects of N=1 type I heterotic duality in four-dimensions', Nucl. Phys. B512 (1998) 221, hepth/9704059; Z. Kakushadze, G. Shiu 'A Chiral $\mathcal{N}=1$ type I vacuum in four dimensions and its heterotic dual', Phys. Rev. D56 (1997) 3686, hep-th/9705163; '4-D chiral $\mathcal{N}=1$ type I vacua with and without D5-branes', Nucl. Phys. B520 (1998) 75, hep-th/9706051. M. Cvetic, M. Plumacher, J. Wang, 'Three family type IIB orientifold string vacua with non-abelian Wilson lines', JHEP 0004 (2000) 004, hep-th/9911021.

[8] G. Zwart, 'Four-dimensional $N=1 Z_{N} \times Z_{M}$ orientifolds', Nucl. Phys. B526 (1998) 378 , hep-th/9708040.

[9] G. Aldazabal, A. Font, L. E. Ibáñez, G. Violero, ' $D=4, N=1$ type IIB orientifolds' Nucl. Phys. B536 (1998) 29, hep-th/9804026.

[10] M. Klein and R. Rabadan, 'D =4, N = 1 orientifolds with vector structure,' hep-th/0007087.

[11] M. Klein and R. Rabadan, ' $\mathrm{Z}(\mathrm{N}) \times \mathrm{Z}(\mathrm{M})$ orientifolds with and without discrete torsion,' hep-th/0008173.

[12] A. Sen, 'Non-BPS states and branes in string theory', hep-th/9904207.

[13] M. R. Gaberdiel, 'Lectures on non-BPS Dirichlet branes', hep-th/0005029.

[14] S. Sugimoto, 'Anomaly cancellations in type I D9 - anti-D9 system and the USp(32) string theory', Prog. Theor. Phys. 102 (1999) 685, hep-th/9905159.

[15] I. Antoniadis, E. Dudas, A. Sagnotti, 'Brane supersymmetry breaking', Phys. Lett. B464 (1999) 38, hep-th/9908023.

[16] G. Aldazabal, A. M. Uranga, 'Tachyon free nonsupersymmetric type IIB orientifolds via brane-antibrane systems', JHEP 9910(1999)024, hep-th/9908072. 
[17] G. Aldazabal, L. E. Ibanez, F. Quevedo, 'Standard-like models with broken supersymmetry from type I string vacua', JHEP 0001 (2000) 031, hep-th/9909172; 'A D-brane alternative to the MSSM', hep-ph/0001083.

[18] C. Angelantonj, I. Antoniadis, G. D'Appollonio, E. Dudas, A. Sagnotti, 'Type I vacua with brane supersymmetry breaking', Nucl. Phys. B572 (2000) 36, hepth/9911081.

[19] C. Angelantonj, R. Blumenhagen, M. R. Gaberdiel, 'Asymmetric orientifolds, brane supersymmetry breaking and non-BPS branes', hep-th/0006033.

[20] G. Aldazabal, L. E. Ibanez, F. Quevedo, A. M. Uranga, 'D-branes at singularities: A Bottom up approach to the string embedding of the standard model', JHEP 0008 (2000) 002, hep-th/0005067.

[21] M. Berkooz, R. G. Leigh, J. Polchinski, J. H. Schwarz, N. Seiberg, E. Witten, "Anomalies, dualities, and topology of $\mathrm{D}=6 \mathrm{~N}=1$ superstring vacua", Nucl. Phys. B475 (1996) 115, hep-th/9605184.

[22] J. Polchinski, 'Tensors from K3 orientifolds', Phys. Rev. D55 (1997) 6423, hepth/9606165.

[23] K. Intriligator, 'RG fixed points in six-dimensions via branes at orbifold singularities', Nucl. Phys. B496 (1997) 177, hep-th/9702038; J. D. Blum, K. Intriligator, "Consistency conditions for branes at orbifold singularities", Nucl. Phys. B506 (1997) 223, hep-th/9705030.

[24] A. Sen, 'BPS D-branes on nonsupersymmetric cycles', JHEP 9812 (1998) 021, hep-th/9812031.

[25] J. Majumder, A. Sen, 'Vortex pair creation on brane-antibrane pair via marginal deformation', JHEP 0006 (2000) 010, hep-th/0003124.

[26] M. R. Gaberdiel, B. Stefanski, Jr, 'Dirichlet branes on orbifolds', Nucl. Phys. B578 (2000) 58, hep-th/9910109.

[27] J. Park, A. M. Uranga, 'A Note on superconformal $\mathcal{N}=2$ theories and orientifolds', Nucl. Phys. B542 (1999) 139, hep-th/9808161.

[28] J. Erler, 'Anomaly cancellation in six-dimensions', J.Math.Phys. 35(1994)1819, hep-th/9304104. 
[29] A. Sagnotti, 'A Note on the Green-Schwarz mechanism in open string theories', Phys. Lett. B294 (1992) 196, hep-th/9210127.

[30] S. Mukhi, N. V. Suryanarayana, D. Tong, 'Brane-antibrane constructions', JHEP 0003 (2000) 015, hep-th/0001066.

[31] L. Dixon, J. A. Harvey, C. Vafa, E. Witten, 'Strings on orbifolds, 2', Nucl. Phys. B274(1986)285.

[32] L. E. Ibáñez, R. Rabadán, A. M. Uranga, 'Anomalous U(1)'s in type I and type IIB $D=4, N=1$ string vacua', Nucl. Phys. B542 (1999) 112, hep-th/9808139.

[33] I. Antoniadis, C. Bachas, E. Dudas, 'Gauge couplings in four-dimensional type I string orbifolds', Nucl. Phys. B560 (1999) 93, hep-th/9906039.

[34] M. Klein, 'Anomaly cancellation in $D=4, \mathcal{N}=1$ orientifolds and linear/chiral multiplet duality', Nucl. Phys. B569 (2000) 362, hep-th/9910143.

[35] J. Park, R. Rabadan, A. M. Uranga, ' $\mathcal{N}=1$ type IIA brane configurations, chirality and T duality', hep-th/9907074; 'Orientifolding the conifold', Nucl. Phys. B570 (2000) 38, hep-th/9907086.

[36] A. M. Uranga, 'A New orientifold of $\mathbf{C}^{\mathbf{2}} / \mathbf{Z}_{\mathbf{N}}$ and six-dimensional RG fixed points', Nucl. Phys. B577 (2000) 73, hep-th/9910155.

[37] A. Hanany, E. Witten, "Type IIB superstrings, BPS monopoles, and threedimensional gauge dynamics", Nucl. Phys. B492 (1997) 152, hep-th/9611230.

[38] K. Landsteiner, E. López, D. A. Lowe, " $\mathrm{N}=2$ supersymmetric gauge theories, branes and orientifolds", Nucl. Phys. B507 (1997) 197, hep-th/9705199.

[39] A. M. Uranga, 'Towards mass deformed $\mathrm{N}=4 \mathrm{SO}(\mathrm{n})$ and $\mathrm{Sp}(\mathrm{k})$ gauge theories from brane configurations', Nucl. Phys. B526 (1998) 241, hep-th/9803054.

[40] E. Witten, 'D-Branes And K-Theory', JHEP 9812 (1998) 019, hep-th/9810188.

[41] M. Frau, L. Gallot, A. Lerda, P. Strigazzi, 'Stable non-BPS D-branes of type I', hep-th/0003022; Nucl. Phys. B564 (2000) 60, hep-th/9903123.

[42] J. Polchinski, 'Dirichlet Branes and Ramond-Ramond charges', Phys. Rev. Lett. 75 (1995) 4724, hep-th/9510017.

[43] D. O'Driscoll, 'General Abelian orientifold models and one loop amplitudes', hepth/9801114. 